\title{
Uma revisão sistemática da epidemiologia e achados histopatológicos de trombos
}

\section{pulmonares na covid-19}

\author{
A systematic review of the epidemiology and histopathological findings of pulmonary thrombi at \\ covid-19
}

Una revisión sistemática de los resultados epidemiológicos e histopatológicos de las trombas pulmonares en covid-19

\section{Resumo}

Alguns dos pacientes afetados pela COVID-19 apresentam trombos. Este estudo teve como objetivo avaliar a associação entre comorbidades da COVID-19 e trombos nos pulmões. Extraíram-se artigos com os descritores "Pathology", "Autopsy", "COVID-19", "SARS-CoV-2" nos bancos de dados: Medical Literature Analysis and Retrieval System Online (MEDLINE/PUBMED), Biblioteca Cochrane, Biblioteca Virtual em Saúde (BVS), Web of Science e Scopus. Os critérios de elegibilidade foram: artigos em inglês, publicados entre 1 de janeiro a 28 de maio de 2020, com casos confirmados de COVID-19, com descrição de necrópsia e análise histopatológica pulmonar. Tabulou-se os dados: idade, sexo, comorbidades, tempo de evolução da doença e achados macro e microscópicos dos pulmões. Vinte artigos foram incluídos, totalizando 119 necrópsias, a maioria deles eram artigos originais ou relatos de caso. Acerca do sexo, 86 eram do masculino, 23 do feminino e 10 eram indeterminados. Setenta e cinco dos indivíduos incluídos apresentaram trombos nos pulmões, dentre esses, 98,7\% apresentaram dano alveolar difuso (DAD). Pacientes que eram ou do sexo masculino ou que apresentavam hipertensão, diabetes ou trombose venosa profunda (TVP) tiveram mais chances de ter trombos nos pulmões. Aproximadamente $70 \%$ dos pacientes analisados tinham entre 60 e 89 anos. A média do tempo de hospitalização foi de 6,4 dias. Em 90,7\% das amostras histológicas pulmonares foram identificados aspectos da DAD. Dos achados macroscópicos dos pulmões, o mais prevalente foi "pulmões pesados", desses, 92,1\% apresentaram trombos. Pacientes internados tiveram uma chance maior de vir a óbito até o $9^{\circ}$ dia de internação. O trabalho identificou casos de trombos em pulmões com maior frequência em indivíduos com TVP, diabetes e hipertensão.

Palavras-chave: Patologia; Autopsia; Histopatológico; Infecções por coronavírus.

\begin{abstract}
Some of the pacients affected by COVID-19 present thrombi. The objective of this study was to assess the association between comorbidities of COVID-19 and thrombi in the lungs. Articles with the descriptors "Pathology", "Autopsy", "COVID-19", "SARS-CoV-2" were extracted from the databases: Medical Literature Analysis and Retrieval System Online (MEDLINE/PUBMED), Cochrane Library, Virtual Health Library (VHL), Web of Science and Scopus. Eligibility criteria were: articles in English, published between January 1 and May 28, 2020, with confirmed cases of COVID-19, with description of necropsy and analysis pulmonary histopathological. The data were tabulated: age, sex, comorbidities, time of disease evolution and macro and microscopic findings of the lungs. Twenty articles were included, totaling 119 necropsies, most of them original articles or case reports Regarding gender, 86 were male, 23 female and 10 were undetermined. Seventy-five of the individuals included had thrombi in the lungs, among these,
\end{abstract}


98.7\% had diffuse alveolar damage (DAD). male or that patients with hypertension, diabetes or deep vein thrombosis (DVT) were more likely to have thrombi in their lungs. Approximately $70 \%$ of the analyzed patients were between 60 and 89 years old. The average length of hospital stay was 6.4 days. In $90.7 \%$ of the pulmonary histological samples, aspects of DAD were identified. Of the macroscopic findings of the lungs, the most prevalent was "heavy lungs", of which $92.1 \%$ had thrombi. Hospitalized patients have a greater chance of dying within the 9th day of hospitalization. The work identified cases of thrombi in the lungs more frequently in individuals with DVT, diabetes and hypertension.

Keywords: Pathology; Autopsy; Histopathology; Coronavirus infections.

\section{Resumen}

Algunos de los pacientes afectados por COVID-19 tienen trombos. Este estudio tuvo como objetivo evaluar la asociación entre comorbilidades de COVID-19 y trombos en los pulmones. Los artículos con los descriptores "Patología", "Autopsia", "COVID-19", "SARS-CoV-2" fueron extraídos de las bases de datos: Medical Literature Analysis and Retrieval System Online (MEDLINE / PUBMED), Cochrane Library, Library Virtual Health (VHL), Web of Science y Scopus Los criterios de elegibilidad fueron: artículos en inglés, publicados entre el 1 de enero y el 28 de mayo de 2020, con casos confirmados de COVID-19, con descripción de necropsia y análisis histopatológico Se tabularon los siguientes datos: edad, sexo, comorbilidades, tiempo de evolución de la enfermedad y hallazgos macroscópicos y microscópicos pulmonares.Se incluyeron 20 artículos, totalizando 119 necropsias, la mayoría originales o reportes de casos, 86 eran masculinos, 23 femeninos y 10 indeterminados. cinco de los individuos incluidos tenían trombos en los pulmones, entre estos, el 98,7\% presentaba daño alveolar difuso (DAD). hipertensión, la diabetes o la trombosis venosa profunda (TVP) tenían más probabilidades de tener trombos en los pulmones. Aproximadamente el $70 \%$ de los pacientes analizados tenían entre 60 y 89 años. La duración media de la estancia hospitalaria fue de 6,4 días. En el 90,7\% de las muestras histológicas pulmonares se identificaron aspectos de DAD. De los hallazgos macroscópicos de los pulmones, el más prevalente fue el de "pulmones pesados", de los cuales el $92,1 \%$ presentaba trombos. Los pacientes hospitalizados tenían una mayor probabilidad de morir al noveno día de hospitalización. El trabajo identificó casos de trombos en los pulmones con mayor frecuencia en personas con TVP, diabetes e hipertensión.

Palabras clave: Patología; Autopsia; Histopatológico; Infecciones por coronavirus.

\section{Introdução}

Em dezembro de 2019 surgiu na China uma série de casos de pneumonia cuja rápida ascensão os colocou no centro dos holofotes mundiais, após sequenciamento e análises identificou-se um novo coronavírus, posteriormente denominado SARS-CoV-2 (Zhu et al., 2019; Huang et al., 2020; Gorbalenya et al., 2020). Filogeneticamente, o vírus se encontra na mesma categoria de Betacoronavírus a que pertencem os vírus responsáveis pelas Síndrome da Angústia Respiratória Aguda (SARS) e Síndrome Respiratória do Oriente Médio (MERS), associadas a prévias epidemias e a características clínicas semelhantes (Tan et al., 2020; Xie \& Chen, 2020).

Apenas seis meses após seu surgimento, a COVID-19 foi responsável por causar uma pandemia de proporções extraordinárias com mais de 10,1 milhões de casos confirmados e cerca de 500 mil mortes por todo o mundo (WHO, 2020).

A sintomatologia mais comum da doença inclui febre, fadiga, tosse seca, mialgia, dispneia e anorexia, sendo náusea e diarreia considerados sintomas atípicos (Wang et al., 2020). Em estudo retrospectivo multicêntrico de coorte, identificou-se que $48 \%$ dos pacientes internados apresentavam comorbidades, sendo hipertensão arterial, diabetes e doença coronariana as mais frequentes (Zhou et al., 2020a). Em um estudo conduzido na China, a forma severa da doença ocorreu em $15,7 \%$ dos pacientes internados avaliados, sendo que destes, $8,1 \%$ foram a óbito, enquanto entre os pacientes com a doença não severa apenas um paciente faleceu, o que representa $0,1 \%$ desta amostra (Guan et al., 2020).

A associação entre D-dímero elevado e maior chance de óbito (Zhou et al., 2020a), além da identificação de trombos e/ou microtrombos em pulmões em achados necroscópicos e avaliações histopatológicas levantaram suspeita de que trombose estaria diretamente relacionada à causa mortis dos pacientes graves (Lax et al., 2020). Adicionalmente, estudos têm encontrado complicações da hipercoagulabilidade no sistema nervoso (Jin et al., 2020), no sistema cardiovascular (Kang et al., 2020), nos rins (Pei et al., 2020) além dos pulmões. 
Sugere-se que a ativação sustentada das vias alternativa e das lectinas do sistema complemento seja responsável pela lesão microvascular trombótica em parte dos pacientes com quadros graves da doença (Magro et al., 2020). Apesar de existir diferença na funcionalidade de aspectos do sistema imune relacionados a sexo e idade (Da Costa et al., 2018), o que poderia contribuir para as diferentes manifestações histológicas pulmonares envolvendo formação de trombos e/ou microtrombos, poucos estudos associam sexo e idade aos achados macro e microscópicos pulmonares de casos fatais de COVID-19.

Assim sendo, a realização de necrópsias de casos fatais de COVID-19, ainda que temida pelo risco de contágio pelo vírus, é necessária para se determinar as condições associadas à doença e sua fisiopatologia, sendo estas indissociáveis (Nunes Duarte et al., 2020; Barth, Xu \& Buja, 2020).

Nesse sentido, o presente estudo tem como objetivo revisar sistematicamente as publicações científicas que descrevem achados macro e microscópicos pulmonares e compreender se existe relação entre a formação de trombos pulmonares e idade, sexo, tempo de evolução da doença e comorbidades em pacientes que foram à óbito com diagnóstico positivo de COVID-19.

\section{Metodologia}

\section{Delineamento}

Essa revisão sistemática de literatura segue as recomendações estabelecidas pelos Principais Itens para Relatar Revisões sistemáticas e Meta-análises (PRISMA) (Moher, Liberati, Tetzlaff \& Altman, 2009). Considerando a limitada disponibilidade de publicações acerca da temática abordada nessa revisão, não foram impostas limitações em relação a viés.

\section{Critérios de elegibilidade}

Foram incluídos artigos publicados em língua inglesa no período de $1^{\circ}$ de janeiro de 2020 a 28 de maio de 2020 que relataram casos confirmados para a infecção por SARS-CoV-2, com descrição de necrópsia e avaliação histopatológica em pelo menos um dos pulmões.

Para avaliar os achados macro e microscópicos pulmonares que evoluíram com ou sem trombos nos pacientes confirmados com COVID-19, os desenhos de estudo elegíveis foram relatos de casos e séries de casos, comunicações rápidas e correspondências considerando as informações a respeito de idade, sexo, tempo de evolução da doença e comorbidades entre pacientes que foram à óbito com diagnóstico positivo de COVID-19. Excluiram-se artigos de revisão e artigos de opinião que não apresentavam dados originais, bem como estudos que relatavam casos com informações incompletas. A avaliação dos estudos foi realizada por três dos autores, os quais fizeram a inclusão de forma independente. Incluíram-se as publicações que obtiveram concordância de julgamento pelos três autores. O processo de seleção dos estudos está descrito em fluxograma (Figura 1). 
Figura 1 - Fluxograma do processo de triagem das publicações.

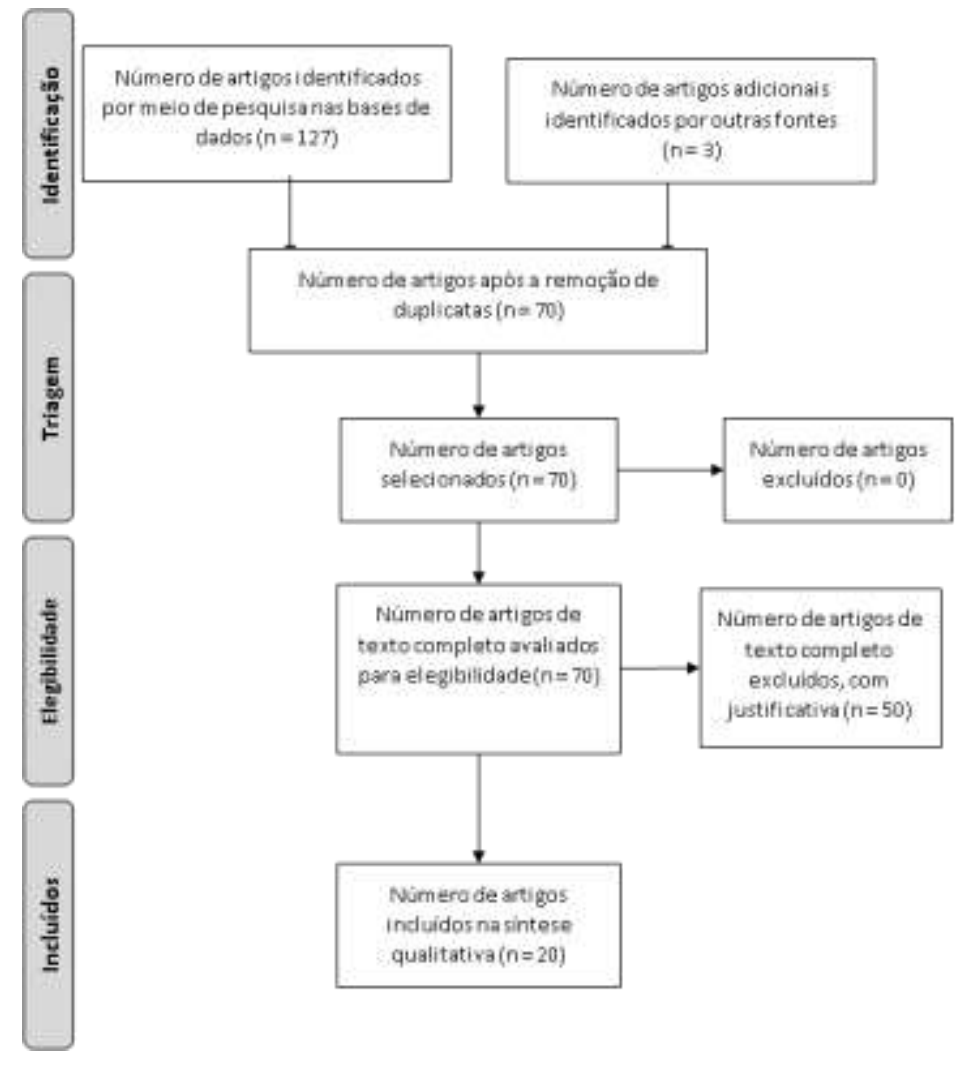

Fonte: Elaborado pelos autores com base no fluxograma PRISMA (Moher, Liberati, Tetzlaff \& Altman, 2009).

\section{Fontes de informação e estratégias de busca}

As buscas bibliográficas foram realizadas em 28 de maio de 2020 nas fontes Medical Literature Analysis and Retrieval System Online (MEDLINE/PUBMED), Biblioteca Cochrane, Biblioteca Virtual em Saúde (BVS), Web of Science e Scopus. Os seguintes descritores de pesquisa foram aplicados: "Pathology", "Autopsy", "COVID-19” e "SARS-CoV-2".

\section{Processo de coleta dos dados}

Os artigos foram selecionados inicialmente por título e resumo. Os textos completos dos artigos relevantes foram examinados quanto aos critérios de inclusão e exclusão.

Dos artigos selecionados, os dados foram coletados em um formulário contendo as seguintes variáveis: 1) nome do artigo; 2) tipo do artigo; 3) número de necrópsias; 4) método de diagnóstico de COVID-19; 5) gênero e idade dos pacientes; 6) comorbidades dos pacientes; 7) tempo de evolução da doença nos pacientes estudados; 8) técnica de necrópsia utilizada; 9) número de amostras coletadas para avaliação histológica; 10) achados da macroscopia; 11) achados da microscopia; e 12) identificação de trombos nos pulmões.

No que se refere aos achados da microscopia, esses foram resumidos segundo sua evolução do Dano Alveolar Difuso (DAD), de acordo com a classificação do website PathologyOutlines.com (Yoshikawa, Fukuoka \& Andrey, 2018).

\section{Resultados}

Foram identificadas 127 publicações a partir da busca virtual nas bases de dados. Após a exclusão dos artigos em duplicata, 67 trabalhos seguiram para avaliação dos critérios de inclusão e exclusão, dos quais 17 respeitaram esses critérios. 
Identificou-se três artigos originais que se enquadravam nos critérios de elegibilidade, mas que não foram previamente encontrados durante as buscas virtuais, esses artigos foram, portanto, adicionados à análise. Assim, incluíramse20 trabalhos, que juntos totalizaram 119 necrópsias. A Figura 1 traz os detalhes do processo de seleção.

\section{Características dos estudos}

As características dos 20 estudos incluídos estão sintetizadas na Tabela 1. Dezessete trabalhos descreveram individualmente as características dos pacientes (sexo, idade e comorbidades) assim como o tempo de evolução da doença até o óbito e os achados macroscópicos e microscópicos pulmonares. Três trabalhos não abordaram de forma individual pelo menos um desses aspectos de estudo, trazendo as informações de forma agrupada por meio de média ou descrição da quantidade de indivíduos com determinado achado macroscópico e microscópico.

A identificação de dois trabalhos que apresentaram, além de necrópsias, biópsias em outros pacientes, levou a exclusão apenas dos pacientes submetidos às biópsias e inclusão das necrópsias nas análises. Cinco trabalhos não especificaram qual o método de diagnóstico utilizado para COVID-19; os demais descreveram que foi realizado por RT-PCR.

Tabela 1 - Características dos estudos incluídos.

\begin{tabular}{|c|c|c|c|c|}
\hline \multicolumn{2}{|r|}{ Publicação } & \multirow{2}{*}{$\begin{array}{l}\text { Tipo de estudo } \\
\text { Correspondência }\end{array}$} & \multirow{2}{*}{$\begin{array}{c}\begin{array}{c}\text { Quantidade de } \\
\text { necrópsias }\end{array} \\
2\end{array}$} & \multirow{2}{*}{$\begin{array}{c}\text { Necrópsias que } \\
\text { apresentaram trombos } \\
0\end{array}$} \\
\hline 1 & Varga et al., 2020* & & & \\
\hline 2 & Karami et al., 2020 & Relato de caso & 1 & 0 \\
\hline 3 & Barton, Duval, Atroberg, Ghosh \& Mukhopadhyay, 2020 & Artigo Original & 2 & 1 \\
\hline 4 & Buja et al., 2020 & Relato de casos & 3 & 1 \\
\hline 5 & Lacy et al., 2020 & $\begin{array}{l}\text { Relato de caso e } \\
\text { revisão }\end{array}$ & 1 & 0 \\
\hline 6 & Ackermann et al., 2020 & Artigo Original & 7 & 7 \\
\hline 7 & Adachi et al., 2020 & Relato de caso & 1 & 0 \\
\hline 8 & Farkash, Wilson \& Jentzen, 2020 & $\begin{array}{c}\text { Rápida } \\
\text { comunicação }\end{array}$ & 1 & 0 \\
\hline 9 & Yan et al., 2020a & Relato de caso & 1 & 0 \\
\hline 10 & Konopka, Wilson \& Myers, 2020 & Relato de caso & 1 & 1 \\
\hline 11 & Aguiar, Lobrinus, Schibler, Fracasso \& Lardi, 2020 & Relato de caso & 1 & 0 \\
\hline 12 & Craver, Huber, Sandomirsky, McKenna, Schieffelin \& Finger, 2020 & Relato de caso & 1 & 0 \\
\hline 13 & Sekulic et al., 2020 & Artigo Original & 2 & 0 \\
\hline 14 & Schweitzer et al., 2020 & $\begin{array}{c}\text { Rápida } \\
\text { comunicação }\end{array}$ & 1 & 0 \\
\hline 15 & Magro et al., $2020^{* *}$ a & Relato de casos & 2 & 1 \\
\hline 16 & Lax et al., 2020 & Artigo Original & 11 & 11 \\
\hline 17 & Menter et al., 2020 & Artigo Original & 21 & 5 \\
\hline 18 & Wichmann et al., $2020^{* * *}$ & Artigo Original & 12 & 5 \\
\hline 19 & Carsana et al., $2020^{* * * a}$ & Artigo Original & 38 & 33 \\
\hline 20 & Fox et al., $2020^{* * * a}$ a & Artigo Original & 10 & 10 \\
\hline & Total de necrópsias & & 119 & 75 \\
\hline
\end{tabular}

* Realizou 2 necrópsias e uma ressecção de intestino, a qual foi excluída da análise.

** Realizou 2 necrópsias e 3 biópsias de pele, as quais foram excluídas da análise.

*** Não abordaram de forma individual pelo menos um dos aspectos de estudo.

${ }^{a}$ Artigos incluídos posteriormente à pesquisa virtual nas bases de dados.

Fonte: Elaborado pelos autores. 


\section{Identificação de trombos}

Das 20 publicações incluídas, 10 indicaram macro e/ou microtrombos em pelo menos uma das necrópsias (Tabela 1).

Do total de 119 necrópsias, 75 (63,0\%) apresentaram pulmões com macro e/ou microtrombos. Entre as 75 necrópsias que identificaram trombos em pulmões, 49 (65,3\%) descreveram apenas microtrombos, sendo um caso associado a trombose venosa profunda (TVP); 26 (34,7\%) indicaram tanto macro quanto microtrombos, sendo quatro destas associadas à TVP.

\section{Características dos pacientes}

As variáveis coletadas dos pacientes se encontram sintetizadas na Tabela 2. Em relação ao sexo, do total de 119 necrópsias, 23 (19,3\%) foram realizadas em pacientes do sexo feminino, 86 (72,3\%) em pacientes do sexo masculino e em 10 necrópsias $(8,4 \%)$ não houve informação do sexo dos pacientes. Dentre os indivíduos do sexo feminino havia uma gestante, compondo $0,8 \%$ da amostra total.

Informações individuais acerca de sexo e avaliação histológica estão disponíveis para 71 pacientes. Do total de 53 homens incluídos nesse grupo, 26 (49,1\%) apresentaram trombo e/ou microtrombo na avaliação histológica. Já entre as 18 mulheres, apenas $6(33,3 \%)$ apresentaram trombo e/ou microtrombo na microscopia. Quatro pacientes do sexo masculino que apresentaram macro e microtrombos e um com microtrombo tiveram associação com trombose venosa profunda. A idade dos pacientes foi dividida em grupos de 10 em 10 anos e analisada a frequência dos pacientes nos intervalos (Gráfico 1).

Gráfico 1 - Modelo de frequência de pacientes por faixas de idade.

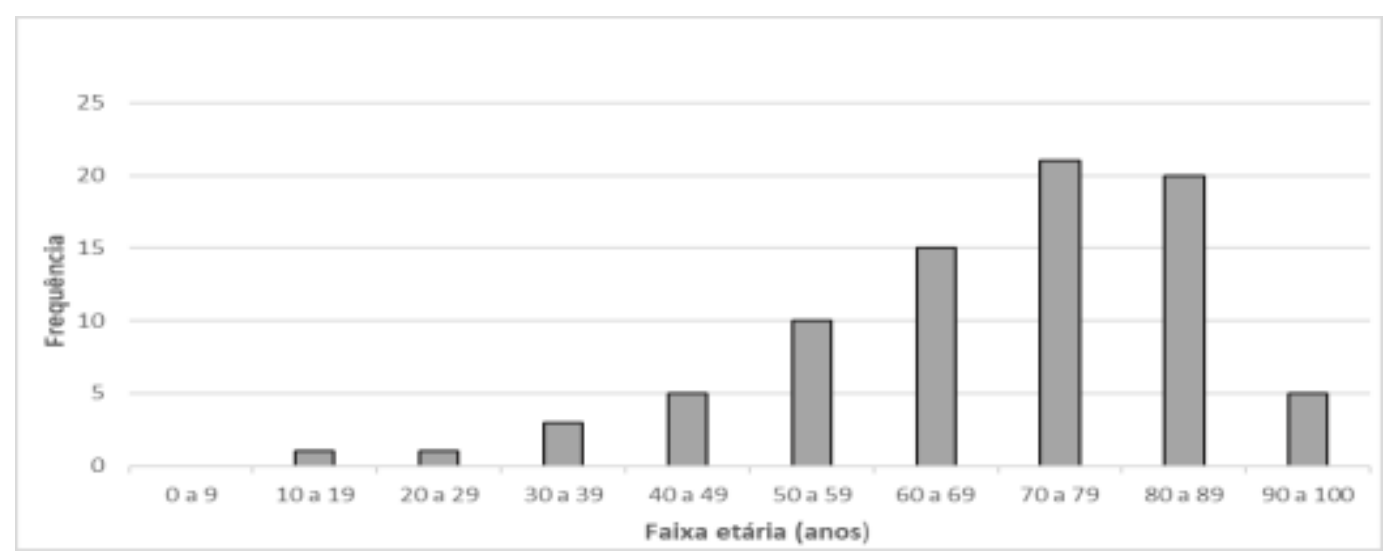

Fonte: Elaborado pelos autores a partir das referências da Tabela 2.

A média dos dados agrupadas em intervalos é de 69,7 anos, o desvio padrão dos dados agrupados foi 1,87.

Pontua-se que um dos artigos (artigo 19 da Tabela 1) não apresentou a idade individualmente dos pacientes, logo, dos 119 pacientes, 38 deles não foram incluídos na análise dos dados de idade da amostra.

Acerca das comorbidades, estão disponíveis os dados de apenas 112 pacientes. Desses, apenas $3(2,7 \%)$ não apresentavam nenhuma comorbidade, sendo que todos os demais possuíam ao menos uma. Dentre os pacientes cujos dados estão disponíveis as comorbidades de maior prevalência são: hipertensão arterial em 69 pacientes (61,6\%), diabetes em 39 $(34,8 \%)$, tabagismo em 16 (14,3\%), doença pulmonar obstrutiva crônica (DPOC) em 10 (8,9\%), doença renal crônica (DRC) em $8(7,1 \%)$, trombose venosa profunda (TVP) esteve presente ou foi parte do histórico de $8(7,1 \%)$, demência em $6(5,3 \%)$, doença arterial coronariana (DAC) em 5 (4,4\%), asma em 4 (3,5\%) e fibrilação atrial em 4 (3,5\%).

Correlacionando a quantidade de indivíduos que foram afetados por cada comorbidade e necrópsias que apresentaram trombos no pulmão, algumas mostraram maior conexão, dentre elas: TVP, 75\% dos pacientes com TVP apresentaram trombos; hipertensão arterial, 68,1\%; diabetes, 64,1\%; DPOC, 60\%; obesidade, 59,3\%; e tabagismo, 56,2\%. 
O tempo de hospitalização dos pacientes até a sua morte foi apresentado em 62 dos 119 pacientes. Dentre esses 62, a média de hospitalização foi de 6,4 dias; o desvio padrão da amostra foi de 4,6; e a mediana foi de 6 (Gráfico 2).

Gráfico 2 - Modelo de frequência de pacientes por tempo de hospitalização.

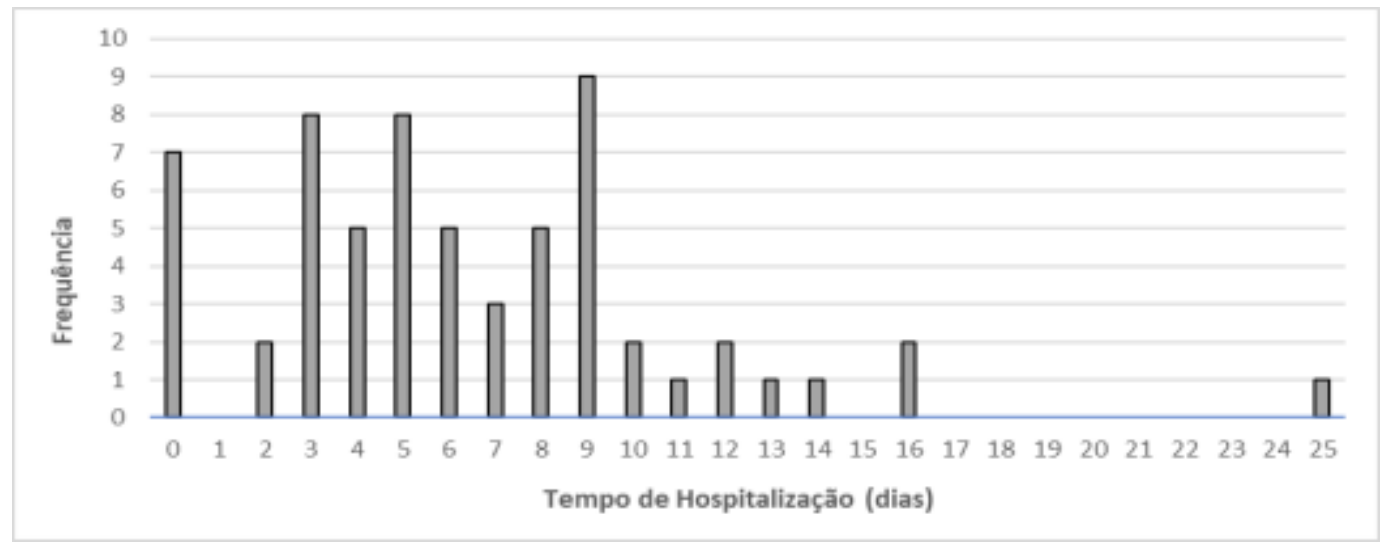

Fonte: Elaborado pelos autores a partir das referências da Tabela 2.

O Gráfico 3 aborda a avaliação de presença ou não de trombos por tempo de hospitalização.

Gráfico 3 - Modelo de frequência de pacientes por tempo de hospitalização relacionando a presença ou não de trombos.

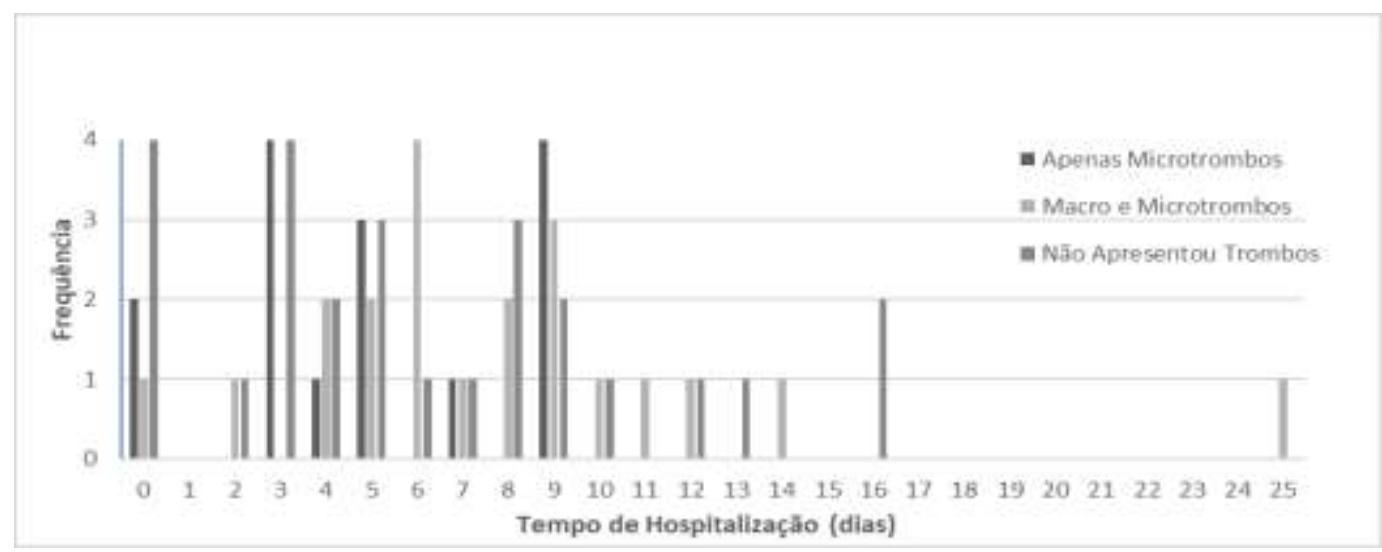

Fonte: Elaborado pelos autores a partir das referências da Tabela 2. 
Tabela 2 - Características dos pacientes e dos achados necroscópicos.

\begin{tabular}{|c|c|c|c|c|c|c|c|c|}
\hline Publicação & Necrópsia & Sexo & Idade (anos) & Comorbidades & $\begin{array}{l}\text { Tempo de evolução da } \\
\text { doença }\end{array}$ & Achados macroscópicos & $\begin{array}{l}\text { Achados } \\
\text { microscópicos }\end{array}$ & Trombos \\
\hline \multirow{2}{*}{1} & 1 & M & 71 & HA, DAC, transplante renal & Hosp.: 8 dias & - & $\begin{array}{l}\text { DAD - Fase } \\
\text { exsudativa }\end{array}$ & NÃO \\
\hline & 2 & $\mathrm{~F}$ & 58 & DM, HA, OBS & Hosp.: 16 dias & - & $\begin{array}{l}\text { Achados condizentes } \\
\text { com SARS }\end{array}$ & NÃO \\
\hline 2 & 3 & $\mathrm{~F}$ & 27 & - & $\begin{array}{l}\text { Sin-Hosp.: } 3 \text { dias } \\
\text { Hosp.: } 3 \text { dias }\end{array}$ & $\begin{array}{c}\text { R-X: OPC, IA } \\
\text { TC: CON, OVF, EP }\end{array}$ & $\begin{array}{l}\text { DAD - Fase } \\
\text { proliferativa }\end{array}$ & NÃO \\
\hline \multirow[b]{2}{*}{3} & 4 & M & 77 & $\begin{array}{l}\text { HA, OBS, TVP, esplenectomia, esteatose, } \\
\text { osteoartrite, hiperplasia } \\
\text { prostática, arterionefroesclerose. }\end{array}$ & Sin-Óbito: 6 dias & $\begin{array}{c}\text { R-X: OPC } \\
\text { Necrópsia: PP, EDM }\end{array}$ & $\begin{array}{l}\text { DAD - Fase } \\
\text { exsudativa }\end{array}$ & SIM (micro) \\
\hline & 5 & M & 42 & $\begin{array}{l}\text { OBS, distrofia muscular, cirrose, esplenomegalia, } \\
\text { ginecomastia, atrofia testicular, } \\
\text { aterosclerose, nefroesclerose, tireoide nodular, rins } \\
\text { com cristais tubulares. }\end{array}$ & Sin-Óbito: 2 dias & $\begin{array}{l}\text { R-X: OPC } \\
\text { TC: CON, OVF } \\
\text { Necrópsia: PP }\end{array}$ & $\begin{array}{l}\text { Sem evidências de } \\
\text { DAD }\end{array}$ & NÃO \\
\hline \multirow{3}{*}{4} & 6 & M & 62 & OBS & Hosp.: 0 dias & Necrópsia: PP & $\begin{array}{l}\text { DAD - Fase } \\
\text { exsudativa }\end{array}$ & NÃO \\
\hline & 7 & M & 34 & OBS, HA, IC, DM II, anemia microcítica & $\begin{array}{l}\text { Sin-Hosp.: } 4 \text { dias } \\
\text { Hosp.: } 10 \text { dias }\end{array}$ & $\begin{array}{c}\text { R-X: OPC } \\
\text { TC: OPC } \\
\text { Necrópsia: CNG, Tr, HMG }\end{array}$ & $\begin{array}{l}\text { DAD - Fase } \\
\text { proliferativa }\end{array}$ & $\begin{array}{l}\text { SIM (macro e } \\
\text { micro) }\end{array}$ \\
\hline & 8 & M & 48 & OBS & Hosp.: 0 dias & Necrópsia: EMP, COL & $\begin{array}{l}\text { DAD - Fase } \\
\text { exsudativa a } \\
\text { proliferativa }\end{array}$ & NÃO \\
\hline 5 & 9 & $\mathrm{~F}$ & 58 & $\begin{array}{l}\text { DM II, OBS, hiperlipidemia, asma, edema de } \\
\text { extremidades inferiores com ulceração }\end{array}$ & Sin-Óbito: 7 dias & Necrópsia: PP, EDM, HMG & $\begin{array}{l}\text { DAD - Fase } \\
\text { exsudativa }\end{array}$ & NÃO \\
\hline \multirow{5}{*}{6} & 10 & $\mathrm{~F}$ & 68 & HA, OBS, esclerose múltipla & Hops.: 9 dias & $\begin{array}{l}\text { TC: OVF } \\
\text { Necrópsia: PP }\end{array}$ & $\begin{array}{l}\text { DAD - Fase } \\
\text { exsudativa a } \\
\text { proliferativa }\end{array}$ & Sim (micro) \\
\hline & 11 & M & 86 & HA, OBS, DM, síndrome mielodisplásica & Hosp.: 5 dias & $\begin{array}{l}\text { TC: CON } \\
\text { Necrópsia: PP }\end{array}$ & $\begin{array}{l}\text { DAD - Fase } \\
\text { exsudativa a } \\
\text { proliferativa }\end{array}$ & Sim (micro) \\
\hline & 12 & M & 96 & HÁ & Hosp.: 3 dias & $\begin{array}{l}\text { TC: CON, OVF } \\
\text { Necrópsia: PP }\end{array}$ & $\begin{array}{l}\text { DAD - Fase } \\
\text { exsudativa a } \\
\text { proliferativa }\end{array}$ & Sim (micro) \\
\hline & 13 & M & 78 & HA, OBS, DM, tabagismo & Hosp.: 3 dias & $\begin{array}{l}\text { TC: CON, OVF } \\
\text { Necrópsia: PP }\end{array}$ & $\begin{array}{l}\text { DAD - Fase } \\
\text { exsudativa a } \\
\text { proliferativa }\end{array}$ & Sim (micro) \\
\hline & 14 & M & 66 & HA, tabagismo & Hosp.: 9 dias & $\begin{array}{l}\text { TC: CON, OVF } \\
\text { Necrópsia: PP }\end{array}$ & $\begin{array}{l}\text { DAD - Fase } \\
\text { exsudativa a } \\
\text { proliferativa }\end{array}$ & Sim (micro) \\
\hline
\end{tabular}


Research, Society and Development, v. 10, n. 8, e0410816983, 2021

(CC BY 4.0) | ISSN 2525-3409 | DOI: http://dx.doi.org/10.33448/rsd-v10i8.16983

\begin{tabular}{|c|c|c|c|c|c|c|c|c|}
\hline & 15 & M & 74 & $\begin{array}{c}\text { HA, DM, CA de próstata com metástase, } \\
\text { tabagismo }\end{array}$ & Hosp.: 3 dias & $\begin{array}{l}\text { TC: CON, OVF } \\
\text { Necrópsia: PP }\end{array}$ & $\begin{array}{l}\text { DAD - Fase } \\
\text { exsudativa a } \\
\text { proliferativa }\end{array}$ & Sim (micro) \\
\hline & 16 & $\mathrm{~F}$ & 81 & HA, tabagismo & Hosp.: 4 dias & $\begin{array}{c}\text { TC: OVF } \\
\text { Necrópsia: PP }\end{array}$ & $\begin{array}{l}\text { DAD - Fase } \\
\text { exsudativa a } \\
\text { proliferativa }\end{array}$ & Sim (micro) \\
\hline 7 & 17 & $\mathrm{~F}$ & 84 & - & $\begin{array}{l}\text { Sin-Hosp.: } 8 \text { dias } \\
\text { Hosp.: } 8 \text { dias }\end{array}$ & $\begin{array}{c}\text { R-X: OPC } \\
\text { TC: CON, OVF } \\
\text { Necrópsia: CON, EP }\end{array}$ & $\begin{array}{l}\text { DAD - Fase } \\
\text { exsudativa }\end{array}$ & Não \\
\hline 8 & 18 & M & 53 & OBS, hiperlipidemia, pós-op. de dissecção aórtica & $\begin{array}{c}\text { Óbito: } 12 \text { dias após cirurgia } \\
\text { para correção de dissecção } \\
\text { aórtica }\end{array}$ & $\mathrm{R}-\mathrm{X}: \mathrm{OPC}$ & $\begin{array}{l}\text { DAD - Fase } \\
\text { exsudativa }\end{array}$ & Não \\
\hline 9 & 19 & $\mathrm{~F}$ & 44 & OBS & $\begin{array}{l}\text { Sin-Hosp.: } 7 \text { dias } \\
\text { Hosp.: } 6 \text { dias }\end{array}$ & Necrópsia: PP, EDM, CON & $\begin{array}{l}\text { DAD - Fase } \\
\text { exsudativa }\end{array}$ & Não \\
\hline 10 & 20 & M & 37 & DM II, asma & $\begin{array}{l}\text { Sin-Hosp.: } 1 \text { dia } \\
\text { Hosp.: } 9 \text { dias }\end{array}$ & $\begin{array}{c}\text { TC: OVF } \\
\text { Necrópsia: CON, PP }\end{array}$ & $\begin{array}{l}\text { DAD - Fase } \\
\text { exsudativa }\end{array}$ & Sim (micro) \\
\hline 11 & 21 & $\mathrm{~F}$ & 31 & OBS mórbida & Sin-Óbito: 7 dias & $\begin{array}{l}\text { TC post-mortem: CON, OVF } \\
\text { Necrópsia: PP, EDM, HMG, } \\
\text { CON, DP }\end{array}$ & $\begin{array}{l}\text { DAD - Fase } \\
\text { exsudativa }\end{array}$ & Não \\
\hline 12 & 22 & M & 17 & - & Sin-Óbito: 2 dias & Necrópsia: PP, CNG & Ausência de DAD & Não \\
\hline \multirow[t]{2}{*}{13} & 23 & M & 81 & $\begin{array}{l}\text { HA, DM, DRC, ICC, DAC (pós-enxerto de } \\
\text { revascularização coronária), FA (marca-passo } \\
\text { biventricular implantado), DAP pós stent ilíaco, } \\
\text { AVC, demência, evidência radiológica de massa } \\
\text { pulmonar esquerda, dislipidemia, gota, tabagismo, } \\
\text { infecções do trato urinário, endarterectomia de } \\
\text { carótida, reparo de hérnia inguinal esquerda e } \\
\text { cirurgia de catarata. }\end{array}$ & $\begin{array}{l}\text { Sin-Hosp.: } 8 \text { dias } \\
\text { Hosp.: } 5 \text { dias }\end{array}$ & $\begin{array}{c}\text { R-X: OPC } \\
\text { TC: OVF, EP } \\
\text { Necrópsia: PP, CNG, ENF }\end{array}$ & $\begin{array}{l}\text { DAD - Fase } \\
\text { exsudativa }\end{array}$ & Não \\
\hline & 24 & M & 54 & HA, DM II, sobrepeso & $\begin{array}{l}\text { Sin-Hosp.: } 2 \text { dias } \\
\text { Hosp.: } 10 \text { dias }\end{array}$ & $\begin{array}{c}\text { R-X: OPC, CON } \\
\text { Necrópsia: PP, EP, CNG }\end{array}$ & $\begin{array}{l}\text { DAD - Fase } \\
\text { exsudativa a } \\
\text { proliferativa }\end{array}$ & Não \\
\hline 14 & 25 & M & 50 & $\begin{array}{l}\text { HIV, infecção pulmonar inespecífica } 5 \text { semanas } \\
\text { antes do óbito }\end{array}$ & Sin-Óbito: 8 dias & Necrópsia: PP & $\begin{array}{l}\text { DAD - Fase } \\
\text { exsudativa }\end{array}$ & Não \\
\hline \multirow[b]{2}{*}{15} & 26 & M & 62 & $\begin{array}{l}\text { DM, IC, DAC, tratamento prévio para hepatite C e } \\
\text { doença renal terminal. }\end{array}$ & Hosp.: horas & $\begin{array}{c}\text { R-X: OPC } \\
\text { Necrópsia: CNG, HMG }\end{array}$ & $\begin{array}{l}\text { Ausência de padrões } \\
\text { de DAD }\end{array}$ & Não \\
\hline & 27 & M & 73 & OBS, pré-DM, tabagismo & $\begin{array}{l}\text { Hosp. (ventilação } \\
\text { mecânica): } 5 \text { dias }\end{array}$ & $\begin{array}{c}\text { R-X: OPC } \\
\text { Necrópsia: CNG, HMG }\end{array}$ & $\begin{array}{l}\text { DAD - Fase } \\
\text { exsudativa a } \\
\text { proliferativa }\end{array}$ & Sim (micro) \\
\hline \multirow{2}{*}{16} & 28 & M & $(80-89) *$ & HA, DM, DAC, doença cerebrovascular & Hosp.: 4 dias & \multirow{2}{*}{$\begin{array}{c}\text { Necrópsia: PP, Tr, ENF (todos) } \\
\text { 9/11: CNG } \\
\text { 1/11: EP }\end{array}$} & $\begin{array}{l}\text { DAD - Fase } \\
\text { exsudativa }\end{array}$ & $\begin{array}{l}\text { Sim (macro e } \\
\text { micro) }\end{array}$ \\
\hline & 29 & M & $(80-89) *$ & HA, DM, doença cerebrovascular & Hosp.: 9 dias & & $\begin{array}{l}\text { DAD - Fase } \\
\text { exsudativa a }\end{array}$ & $\begin{array}{l}\text { Sim (macro e } \\
\text { micro) }\end{array}$ \\
\hline
\end{tabular}


Research, Society and Development, v. 10, n. 8, e0410816983, 2021

(CC BY 4.0) | ISSN 2525-3409 | DOI: http://dx.doi.org/10.33448/rsd-v10i8.16983

\begin{tabular}{|c|c|c|c|c|c|c|c|c|}
\hline & & & & & & & proliferativa & \\
\hline & 30 & $\mathrm{~F}$ & $(80-89) *$ & HA, doença cerebrovascular & Hosp.: 6 dias & & $\begin{array}{l}\text { DAD - Fase } \\
\text { exsudativa a } \\
\text { proliferativa }\end{array}$ & $\begin{array}{l}\text { Sim (macro e } \\
\text { micro) }\end{array}$ \\
\hline & 31 & M & $(80-89) *$ & HA, DAC, doença maligna prévia & $\begin{array}{l}\text { Sin-Hosp.: } 4 \text { dias } \\
\text { Hosp: } 7 \text { dias }\end{array}$ & & $\begin{array}{l}\text { DAD - Fase } \\
\text { exsudativa a } \\
\text { proliferativa }\end{array}$ & $\begin{array}{l}\text { Sim (macro e } \\
\text { micro) }\end{array}$ \\
\hline & 32 & M & $(80-89) *$ & Doença maligna prévia & $\begin{array}{l}\text { Sin-Hosp.: } 5 \text { dias } \\
\text { Hosp.: } 6 \text { dias }\end{array}$ & & $\begin{array}{l}\text { DAD - Fase } \\
\text { exsudativa a } \\
\text { proliferativa }\end{array}$ & $\begin{array}{l}\text { Sim (macro e } \\
\text { micro) }\end{array}$ \\
\hline & 33 & M & $(80-89) *$ & HA, DAC, DPOC & $\begin{array}{l}\text { Sin-Hosp.: } 2 \text { dias } \\
\text { Hosp.: } 8 \text { dias }\end{array}$ & & $\begin{array}{l}\text { DAD - Fase } \\
\text { exsudativa }\end{array}$ & $\begin{array}{l}\text { Sim (macro e } \\
\text { micro) }\end{array}$ \\
\hline & 34 & $\mathrm{~F}$ & $(80-89) *$ & HA, DM, DPOC, demência & Hosp.: 4 dias & & $\begin{array}{l}\text { DAD - Fase } \\
\text { exsudativa a } \\
\text { proliferativa }\end{array}$ & $\begin{array}{l}\text { Sim (macro e } \\
\text { micro) }\end{array}$ \\
\hline & 35 & $\mathrm{~F}$ & $(70-79) *$ & HA, DM, demência & $\begin{array}{l}\text { Sin-Hosp.: } 1 \text { dia } \\
\text { Hosp: } 5 \text { dias }\end{array}$ & & $\begin{array}{l}\text { DAD - Fase } \\
\text { exsudativa a } \\
\text { proliferativa }\end{array}$ & $\begin{array}{l}\text { Sim (macro e } \\
\text { micro) }\end{array}$ \\
\hline & 36 & M & $(60-69) *$ & Demência & Hosp.: 6 dias & & $\begin{array}{l}\text { DAD - Fase } \\
\text { exsudativa a } \\
\text { proliferativa }\end{array}$ & $\begin{array}{l}\text { Sim (macro e } \\
\text { micro) }\end{array}$ \\
\hline & 37 & M & $(90-99) *$ & HA, DM, demência & $\begin{array}{l}\text { Sin-Hosp.: } 1 \text { dia } \\
\text { Hosp.: } 9 \text { dias }\end{array}$ & & $\begin{array}{l}\text { DAD - Fase } \\
\text { exsudativa a } \\
\text { proliferativa }\end{array}$ & $\begin{array}{l}\text { Sim (macro e } \\
\text { micro) }\end{array}$ \\
\hline & 38 & M & $(70-79) *$ & HÁ & $\begin{array}{l}\text { Sin-Hosp.: } 7 \text { dias } \\
\text { Hosp.: } 11 \text { dias }\end{array}$ & & $\begin{array}{l}\text { DAD - Fase } \\
\text { exsudativa }\end{array}$ & $\begin{array}{l}\text { Sim (macro e } \\
\text { micro) }\end{array}$ \\
\hline \multirow{7}{*}{17} & 39 & $\mathrm{~F}$ & 68 & HA, OBS, aterosclerose, esclerose múltipla & Hosp.: 9 dias & Achados radiológicos: OVF & $\begin{array}{l}\text { DAD - Fase } \\
\text { exsudativa }\end{array}$ & Sim (micro) \\
\hline & 40 & M & 86 & $\begin{array}{l}\text { HA, DM II, CA de próstata, apneia do sono, } \\
\text { dislipidemia }\end{array}$ & Hosp.: 5 dias & Achados radiológicos: CON & $\begin{array}{l}\text { DAD - Fase } \\
\text { exsudativa }\end{array}$ & Não \\
\hline & 41 & M & 96 & $\begin{array}{l}\text { HA, DAC, doença de Parkinson, demência, infarto } \\
\text { do miocárdio }\end{array}$ & Hosp.: 3 dias & $\begin{array}{l}\text { Achados radiológicos: OVF, } \\
\text { CON }\end{array}$ & $\begin{array}{l}\text { Sem evidências de } \\
\text { DAD }\end{array}$ & Sim (micro) \\
\hline & 42 & M & 78 & $\begin{array}{l}\text { HA, OBS, DM II, DRC, DAC, FA, hiperuricemia, } \\
\text { apneia do sono, tabagismo, dislipidemia, } \\
\text { reconstrução de valva aórtica }\end{array}$ & Hosp.: 3 dias & $\begin{array}{l}\text { Achados radiológicos: OVF, } \\
\text { CON }\end{array}$ & $\begin{array}{l}\text { DAD - Fase } \\
\text { exsudativa }\end{array}$ & Não \\
\hline & 43 & M & 66 & HA, tabagismo & Hosp.: 9 dias & $\begin{array}{l}\text { Achados radiológicos: OVF, } \\
\text { CON }\end{array}$ & $\begin{array}{l}\text { DAD - Fase } \\
\text { exsudativa }\end{array}$ & Não \\
\hline & 44 & M & 74 & $\begin{array}{l}\text { HA, DM II, DRC, DAC, DPOC, CA prostático } \\
\text { metastático, trombofilia genética, tabagismo, } \\
\text { ressecção pulmonar em cunha }\end{array}$ & Hosp.: 3 dias & $\begin{array}{l}\text { Achados radiológicos: OVF, } \\
\text { CON }\end{array}$ & $\begin{array}{l}\text { DAD - Fase } \\
\text { exsudativa }\end{array}$ & Não \\
\hline & 45 & $\mathrm{~F}$ & 81 & HA, DAC, doença arterial periférica, tabagismo & Hosp.: 4 dias & Achados radiológicos: OVF & $\begin{array}{l}\text { DAD - Fase } \\
\text { exsudativa }\end{array}$ & Não \\
\hline
\end{tabular}


Research, Society and Development, v. 10, n. 8, e0410816983, 2021

(CC BY 4.0) | ISSN 2525-3409 | DOI: http://dx.doi.org/10.33448/rsd-v10i8.16983

\begin{tabular}{|c|c|c|c|c|c|c|c|}
\hline 46 & M & 71 & $\begin{array}{l}\text { DAC, doença arterial periférica, aneurisma de } \\
\text { aorta, valvulopatia, by-pass duplo }\end{array}$ & Hosp.: 0 dias & $\begin{array}{l}\text { Achados radiológicos: OVF, } \\
\text { CON }\end{array}$ & $\begin{array}{l}\text { DAD - Fase } \\
\text { exsudativa }\end{array}$ & Sim (micro) \\
\hline 47 & M & 88 & $\begin{array}{c}\text { HA, IC, DAC, } \\
\text { FA, Macroglobulinemia de Waldeström, basalioma }\end{array}$ & Hosp.: 2 dias & Achados radiológicos: OVF & $\begin{array}{l}\text { DAD - Fase } \\
\text { exsudativa }\end{array}$ & Não \\
\hline 48 & M & 85 & $\begin{array}{c}\text { FA, cardiopatia hipertensiva, tabagismo, hipertrofia } \\
\text { cardíaca excêntrica com fração de ejeção } \\
\text { hiperdinâmica }\end{array}$ & Hosp.: 5 dias & Achados radiológicos: OVF & $\begin{array}{l}\text { DAD - Fase } \\
\text { exsudativa }\end{array}$ & Sim (micro) \\
\hline 49 & M & 58 & HA, OBS, aterosclerose & Hosp.: 7 dias & $\begin{array}{l}\text { Achados radiológicos: OVF, } \\
\text { CON }\end{array}$ & $\begin{array}{l}\text { DAD - Fase } \\
\text { exsudativa }\end{array}$ & Sim (micro) \\
\hline 50 & M & 75 & HA, DM II, DAC, dislipidemia, apneia do sono & Hosp.: 3 dias & $\begin{array}{l}\text { Achados radiológicos: OVF, } \\
\text { CON }\end{array}$ & $\begin{array}{l}\text { Sem evidências de } \\
\text { DAD }\end{array}$ & Não \\
\hline 51 & M & 53 & $\begin{array}{l}\text { HA, OBS, DM II, HIV, cirrose hepática, apneia do } \\
\text { sono }\end{array}$ & Hosp.: 8 dias & Achados radiológicos: $\mathrm{CON}$ & $\begin{array}{l}\text { DAD - Fase } \\
\text { exsudativa a } \\
\text { proliferativa }\end{array}$ & Não \\
\hline 52 & $\mathrm{~F}$ & 94 & $\begin{array}{l}\text { HA, FA, síndrome da taqui- } \\
\text { bradicardia, valvulopatia, demência }\end{array}$ & Hosp.: 0 dias & - & $\begin{array}{l}\text { Sem evidências de } \\
\text { DAD }\end{array}$ & Não \\
\hline 53 & M & 89 & $\begin{array}{l}\text { HA, DM II, DAC, valvulopatia, bloqueio átrio- } \\
\text { ventricular de III grau, dislipidemia, tabagismo }\end{array}$ & Hosp.: 5 dias & Achados radiológicos: $\mathrm{CON}$ & $\begin{array}{l}\text { DAD - Fase } \\
\text { exsudativa a } \\
\text { proliferativa }\end{array}$ & Não \\
\hline 54 & $\mathrm{~F}$ & 61 & HA, OBS, DM II & Hosp.: 9 dias & $\begin{array}{l}\text { Achados radiológicos: OVF, } \\
\text { CON }\end{array}$ & $\begin{array}{l}\text { DAD - Fase } \\
\text { exsudativa a } \\
\text { proliferativa }\end{array}$ & Não \\
\hline 55 & M & 72 & HA, apneia do sono, gota, tabagismo & Hosp.: 12 dias & - & $\begin{array}{l}\text { DAD - Fase } \\
\text { proliferativa }\end{array}$ & Não \\
\hline 56 & M & 79 & $\begin{array}{l}\text { HA, DAC, dislipidemia, doença de Parkinson e de } \\
\text { Alzheimer }\end{array}$ & Hosp.: 16 dias & Achados radiológicos: $\mathrm{CON}$ & $\begin{array}{l}\text { Sem evidências de } \\
\text { DAD }\end{array}$ & Não \\
\hline 57 & M & 65 & $\begin{array}{l}\text { HA, DAC, DPOC, apneia do sono, distúrbio } \\
\text { bipolar, tabagismo }\end{array}$ & Hosp.: 7 dias & $\begin{array}{l}\text { Achados radiológicos: OVF, } \\
\text { CON }\end{array}$ & $\begin{array}{l}\text { DAD - Fase } \\
\text { exsudativa }\end{array}$ & Não \\
\hline 58 & M & 71 & HA, OBS. DPOC, gota, dislipidemia & Hosp.: 4 dias & Achados radiológicos: $\mathrm{CON}$ & $\begin{array}{l}\text { DAD - Fase } \\
\text { exsudativa a } \\
\text { proliferativa }\end{array}$ & Não \\
\hline 59 & M & 96 & HA, DAC, demência & Hosp.: 13 dias & - & $\begin{array}{l}\text { DAD - Fase } \\
\text { exsudativa a } \\
\text { proliferativa }\end{array}$ & Não \\
\hline & & & & & & & \\
\hline 60 & M & 52 & OBS & Hop.: 0 dias & $\begin{array}{l}\text { TC post-mortem: CON } \\
\text { Necrópsia: EMB, PNM }\end{array}$ & $\begin{array}{c}\text { DAD - Fase } \\
\text { exsudativa a } \\
\text { proliferativa. Fibrose } \\
\text { discreta }\end{array}$ & $\begin{array}{l}\text { Sim (micro e } \\
\text { macro - } \\
\text { associação } \\
\text { com TVP) }\end{array}$ \\
\hline 61 & M & 70 & $\begin{array}{l}\text { DRC, DCC, doença arterial periférica, doença de } \\
\text { Parkinson }\end{array}$ & $\begin{array}{l}\text { Tempo } \\
\text { de hosp. indeterminado }\end{array}$ & $\begin{array}{l}\text { Necrópsia: PNM, bronquite } \\
\text { purulenta }\end{array}$ & $\begin{array}{l}\text { DAD - Fase } \\
\text { exsudativa a }\end{array}$ & Não \\
\hline
\end{tabular}


Research, Society and Development, v. 10, n. 8, e0410816983, 2021

(CC BY 4.0) | ISSN 2525-3409 | DOI: http://dx.doi.org/10.33448/rsd-v10i8.16983

\begin{tabular}{|c|c|c|c|c|c|c|c|c|}
\hline & & & & & & & proliferativa & \\
\hline & 62 & M & 71 & HA, tabagismo, pneumopatia granulomatosa & $\begin{array}{c}\text { Tempo } \\
\text { de hosp. indeterminado }\end{array}$ & $\begin{array}{l}\text { TC post-mortem: CON, ENF } \\
\text { Necrópsia: EMB, PNM }\end{array}$ & $\begin{array}{l}\text { DAD - Fase } \\
\text { exsudativa a } \\
\text { proliferativa }\end{array}$ & $\begin{array}{l}\text { Sim (micro e } \\
\text { macro - } \\
\text { associação } \\
\text { com TVP) }\end{array}$ \\
\hline & 63 & M & 63 & OBS, DM II, asma brônquica & $\begin{array}{c}\text { Tempo } \\
\text { de hosp. indeterminado }\end{array}$ & Necrópsia: EMB, PNM & $\begin{array}{l}\text { DAD - Fase } \\
\text { exsudativa a } \\
\text { proliferativa }\end{array}$ & $\begin{array}{l}\text { Sim (micro e } \\
\text { macro - } \\
\text { associação } \\
\text { com TVP) }\end{array}$ \\
\hline & 64 & M & 66 & DCC & Hosp..: 0 & $\begin{array}{l}\text { TC post-mortem: CON, PR } \\
\text { Necrópsia: } \mathrm{PNM}\end{array}$ & $\begin{array}{l}\text { DAD - Fase } \\
\text { exsudativa a } \\
\text { proliferativa }\end{array}$ & $\begin{array}{l}\text { Sim (micro- } \\
\text { associação } \\
\text { com TVP) }\end{array}$ \\
\hline & 65 & $\mathrm{~F}$ & 54 & Demência, epilepsia, trissomia do 21 & $\begin{array}{c}\text { Tempo } \\
\text { de hosp. indeterminado }\end{array}$ & $\begin{array}{c}\text { TC post-mortem: CON, OVF, PR } \\
\text { Necrópsia: PNM }\end{array}$ & Ausência de DAD & Não \\
\hline & 66 & $\mathrm{~F}$ & 75 & FA, DCC, tabagismo & $\begin{array}{c}\text { Tempo } \\
\text { de hosp. indeterminado }\end{array}$ & $\begin{array}{c}\text { TC post-mortem: CON, PR } \\
\text { Necrópsia: ENF, PNM }\end{array}$ & $\begin{array}{l}\text { DAD - Fase } \\
\text { exsudativa a } \\
\text { proliferativa }\end{array}$ & Não \\
\hline & 67 & M & 82 & DM II, DCC, Doença de Parkinson & $\begin{array}{c}\text { Tempo } \\
\text { de hosp. indeterminado }\end{array}$ & $\begin{array}{c}\text { TC post-mortem: CON, EP, ENF, } \\
\text { PR } \\
\text { Necrópsia: ENF, PNM }\end{array}$ & Ausência de DAD & $\begin{array}{l}\text { Não } \\
\text { (associação } \\
\text { com TVP) }\end{array}$ \\
\hline & 68 & $\mathrm{~F}$ & 87 & $\begin{array}{c}\text { DRC, DPOC, DCC, CA de pulmão de células não } \\
\text { pequenas }\end{array}$ & $\begin{array}{c}\text { Tempo } \\
\text { de hosp. indeterminado }\end{array}$ & $\begin{array}{c}\text { TC post-mortem: CON, ENF, PR, } \\
\text { TU } \\
\text { Necrópsia: ENF, PNM, TU }\end{array}$ & Ausência de DAD & Não \\
\hline & 69 & M & 84 & HA, DM II, colite ulcerativa & $\begin{array}{c}\text { Tempo } \\
\text { de hosp. indeterminado }\end{array}$ & $\begin{array}{c}\text { TC post-mortem: CON, OVF, EP, } \\
\text { PR } \\
\text { Necrópsia: ENF, PNM }\end{array}$ & $\begin{array}{l}\text { Ausência de DAD. } \\
\text { Fibrose }\end{array}$ & Não \\
\hline & 70 & M & 85 & HA, FA, DCC, asma brônquica & $\begin{array}{c}\text { Tempo } \\
\text { de hosp. indeterminado }\end{array}$ & $\begin{array}{l}\text { TC post-mortem: CON, OVF, EP } \\
\text { Necrópsia: ENF, EMB, PNM }\end{array}$ & $\begin{array}{l}\text { DAD - Fase } \\
\text { exsudativa a } \\
\text { proliferativa }\end{array}$ & $\begin{array}{c}\text { Não } \\
\text { (associação } \\
\text { com TVP) }\end{array}$ \\
\hline & 71 & M & 76 & OBS & $\begin{array}{c}\text { Tempo } \\
\text { de hosp. indeterminado }\end{array}$ & $\begin{array}{c}\text { TC post-mortem: EP } \\
\text { Necrópsia: ENF, EMB, PNM, } \\
\text { traqueobronquite purulenta }\end{array}$ & $\begin{array}{c}\text { DAD - Fase } \\
\text { exsudativa a } \\
\text { proliferativa. Fibrose }\end{array}$ & $\begin{array}{l}\text { Sim (micro e } \\
\text { macro - } \\
\text { associação } \\
\text { com TVP) }\end{array}$ \\
\hline 19 & $72-109$ & $\begin{array}{l}33 \mathrm{M} \\
5 \mathrm{~F}\end{array}$ & $69(32-86)$ & $\begin{array}{c}\text { Dados de apenas } 31 \text { pacientes: } \\
18 \text { HA (58\%) } \\
9 \text { DM }(29 \%) \\
4 \text { neoplasias passadas }(13 \%) \\
11 \text { problemas cardiovasculares }(35 \%) \\
3 \text { DPOC }(10 \%)\end{array}$ & $\begin{array}{c}\text { Sin-Óbito (média) } 16 \text { dias } \\
\text { (5-31) DP=6 } \\
\text { UTI/Subintensivo (média): } \\
7 \text { dias (1-23) DP=6 }\end{array}$ & $\begin{array}{c}\text { Achados } \\
\text { radiológicos (todos): PNM } \\
\text { Necrópsia (todos): PP, EDM, } \\
\text { CNG }\end{array}$ & $\begin{array}{l}\text { Todos: DAD - Fase } \\
\text { exsudativa ou } \\
\text { proliferativa. }\end{array}$ & $\begin{array}{l}\text { Sim (micro } \\
33 / 38)\end{array}$ \\
\hline 20 & 110 & $\begin{array}{l}\text { Não } \\
\text { informa }\end{array}$ & 44 & HA, DM, DRC, OBS & $\begin{array}{l}\text { Sin-Óbito: } 11 \text { dias } \\
\text { Hosp.: } 8 \text { dias } \\
\text { Ventilação: } 6 \text { dias }\end{array}$ & $\begin{array}{l}\text { Necrópsia: 10/10 Tr, EDM. 9/10 } \\
\text { PP, HMG. 1/10 CON }\end{array}$ & $\begin{array}{l}\text { Todos: DAD } \\
\text { 2: Fase exsudativa } \\
\text { 7: Transição para }\end{array}$ & $\begin{array}{l}\text { Sim (macro } \\
\text { micro } 10 / 10)\end{array}$ \\
\hline
\end{tabular}


Research, Society and Development, v. 10, n. 8, e0410816983, 2021

(CC BY 4.0) | ISSN 2525-3409 | DOI: http://dx.doi.org/10.33448/rsd-v10i8.16983

\begin{tabular}{|c|c|c|c|c|}
\hline 111 & 44 & HA, DM, OBS & $\begin{array}{l}\text { Sin-Óbito: } 11 \text { dias } \\
\text { Hosp.: } 5 \text { dias } \\
\text { Ventilação: } 2 \text { dias }\end{array}$ & \multirow[t]{9}{*}{$\begin{array}{l}\text { Fase proliferativa } \\
\text { 1: Fase proliferativa a } \\
\text { fibrótica }\end{array}$} \\
\hline 112 & 63 & HA, tireoidectomia, OBS & $\begin{array}{l}\text { Sin-Óbito: } 32 \text { dias } \\
\text { Hosp.: } 25 \text { dias } \\
\text { Ventilação: } 25 \text { dias }\end{array}$ & \\
\hline 113 & 76 & HA, DM, artrite reumatoide, OBS & $\begin{array}{l}\text { Sin-Óbito: } 9 \text { dias } \\
\text { Hosp.: } 6 \text { dias } \\
\text { Ventilação: } 6 \text { dias }\end{array}$ & \\
\hline 114 & 68 & DM, OBS & $\begin{array}{l}\text { Sin-Óbito: } 10 \text { dias } \\
\text { Hosp.: } 9 \text { dias } \\
\text { Ventilação: } 9 \text { dias }\end{array}$ & \\
\hline 115 & 78 & OBS & Sin-Óbito: 7 dias & \\
\hline 116 & 53 & HA, poliomiosite, apneia do sono obstrutiva & Sin-Óbito: 1 dia & \\
\hline 117 & 60 & $\begin{array}{c}\text { HA, FA, DPOC, parada cardíaca pós-intubação, } \\
\text { OBS }\end{array}$ & $\begin{array}{l}\text { Sin-Óbito: } 17 \text { dias } \\
\text { Hosp.: } 14 \text { dias } \\
\text { Ventilação: } 14 \text { dias }\end{array}$ & \\
\hline 118 & 66 & HA & $\begin{array}{l}\text { Sin-Óbito: } 14 \text { dias } \\
\text { Hosp.: } 12 \text { dias } \\
\text { Ventilação: } 12 \text { dias }\end{array}$ & \\
\hline 119 & 78 & DM, doença renal terminal, OBS & $\begin{array}{l}\text { Sin-Óbito: } 4 \text { dias } \\
\text { Hosp.: } 2 \text { dias } \\
\text { Ventilação: } 2 \text { dias }\end{array}$ & \\
\hline
\end{tabular}

Fonte: Elaborado pelos autores a partir das referências da Tabela 1.

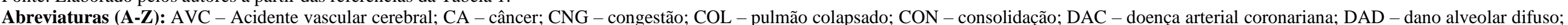

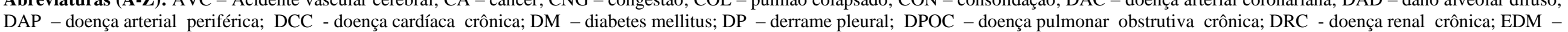

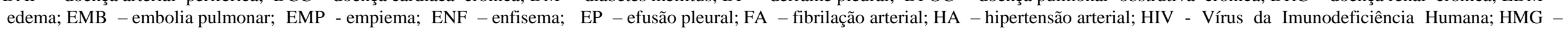

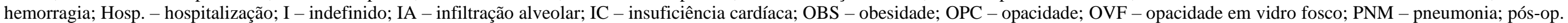

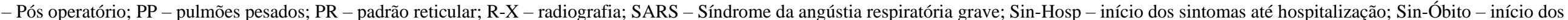
sintomas até o óbito; TC - tomografia computadorizada; $\mathrm{Tr}$ - trombo; TU - tumor; TVP - trombose venosa profunda.

*Artigo optou por apresentar apenas a faixa etária a que pertenciam os indivíduos para garantir o anonimato. 


\section{Achados macroscópicos dos pulmões}

Os achados macroscópicos foram divididos em dois segmentos de análise, são eles: achados radiológicos pulmonares e achados da análise macroscópica de necrópsia dos pulmões. A Tabela 2 sintetiza na aba de "achados macroscópicos" apenas os achados mais relevantes dos artigos.

Das 119 necrópsias realizadas: 94 (79\%) descreveram achados macroscópicos dos pulmões, 85 (71,4\%) exibiram achados radiológicos e $5(4,2 \%)$ não citaram quaisquer achados macroscópicos.

A maior parte dos achados radiológicos dos pulmões foram fruto de R-X ou TC (apenas os artigos 17 e 19 não discriminaram o tipo de exame), sendo que as manifestações de maior incidência entre os 85 indivíduos analisados foram: pneumonia em 38 (44,7\%) pacientes, consolidação em 33 (38,8\%), opacidade em vidro fosco em 28 (32,9\%), opacidade em 10 $(8,4 \%)$, efusão pleural em $6(5 \%)$ e padrão reticular em $6(5 \%)$. Destaca-se que os achados de pneumonia mencionados são todos pertencentes ao artigo 18 .

Correlacionando os achados radiológicos com o aparecimento de trombos, percebeu-se que alguns achados se relacionavam mais fortemente com a presença de trombos: dentre os que apresentaram pneumonia, em $86,8 \%$ verificou-se trombos; opacidade em vidro fosco em 42,8\%; consolidação em 33,3\%; e opacidade em $30 \%$.

Já tratando-se dos achados macroscópicos pulmonares visualizados no momento da necrópsia, as características mais comuns dentre os pacientes descritos incluíram: pulmões pesados em 76 (80,8\%), congestão em 53 (56,4\%), edema em 52 (55,3\%), trombos em $22(23,4 \%)$, enfisema em 18 (19,1\%), hemorragia em $14(14,9 \%)$, pneumonia em 12 (12,8\%), embolia em $5(5,3 \%)$ e consolidação em $5(5,3 \%)$.

Correlacionando os achados macroscópicos com o aparecimento de trombos, percebeu-se que, dentre os que apresentaram pulmões pesados, em 92,1\% verificou-se trombos; edema em 84,6\%; congestão em 83\%; hemorragia em 78,6\%; e enfisema em $66,7 \%$.

\section{Achados microscópicos dos pulmões}

Em relação aos achados microscópicos, das 119 análises histológicas de pulmões, identificou-se aspectos condizentes com DAD em 108 (90,7\%). Entre as análises que identificaram DAD, 27 (25,0\%) referem-se à fase exsudativa, 4 (3,7\%) à fase proliferativa, $38(35,2 \%)$, a ambas as fases proliferativa e exsudativa. Outros 38 pacientes $(35,2 \%)$ que apresentaram DAD na microscopia pulmonar foram descritos de forma conjunta demonstrando achados de fase exsudativa ou proliferativa. Uma das necrópsias abordou achado histológico compatível com síndrome da angústia respiratória grave, porém não descreveu a fase. Três análises histológicas identificaram a presença de fibrose, sendo que duas delas também observaram DAD em fase exsudativa a proliferativa e uma delas não possuía aspecto característico de DAD.

A Tabela 3 aborda os tipos de trombos identificados nas necrópsias em relação à fase de dano alveolar difuso (DAD). 
Tabela 3 - Tipo de trombo em relação à fase de dano alveolar difuso (DAD) identificados nas necrópsias.

\begin{tabular}{lcccccc}
\hline Fase / Tipo de trombo & Exsudativa & Proliferativa & Exs. a Prol. & Aus. DAD & $\begin{array}{c}\text { Exs. ou } \\
\text { Prol. }\end{array}$ & $\begin{array}{c}\text { Prol. a } \\
\text { Fibr. }\end{array}$ \\
\hline Microtrombos & 6 & 0 & 9 & 1 & 33 & 0 \\
Macro e microtrombos & 5 & 1 & 19 & 0 & 0 & 1 \\
$\begin{array}{l}\text { Macro e microtrombos } \\
\text { associados a TVP }\end{array}$ & 0 & 0 & 4 & 0 & 0 & 0 \\
\hline
\end{tabular}

Fonte: Elaborado pelos autores a partir das referências da Tabela 1.

Exs. a Prol - Exsudativa a proliferativa; Aus. DAD - Ausência de DAD; Exs. ou Prol. - Exsudativa ou proliferativa; Prol. a Fibr. Proliferativa a fibrose; TVP - Trombose venosa profunda.

\section{Discussão}

A identificação de macro e/ou microtrombos em $63 \%$ das necrópsias analisadas no presente estudo condiz com a informação de que há elevada prevalência de eventos trombóticos na COVID-19, cuja causa sugere-se estar relacionada a complexas interações entre mecanismos imunológicos do indivíduo e o sistema de coagulação (Iba, Levy, Levi, Connors \& Thachil, 2020). Além disso, mesmo na ausência de macro e/ou microtrombos observou-se, à microscopia eletrônica, fios de fibrina precipitados e neutrófilos em capilares alveolares, assim como maior deposição de fibrina em espaços alveolares (Buja et al., 2020). Os achados de um estudo demonstraram que a formação de microtrombos em capilares alveolares é nove vezes mais prevalente na COVID-19 do que na Influenza A (H1N1) (Ackermann et al., 2020). Nesse sentido, percebe-se que o estado pró-trombótico identificado na COVID-19 está associado à severidade da enfermidade.

O estudo sistematizado acerca de causas bem conhecidas da coagulopatia indica que a COVID-19 apresenta um padrão único de coagulopatia, nos mostrando que a causa da formação de trombos na COVID-19 não é plenamente conhecida (Iba, Levy, Levi, Connors \& Thachil, 2020).

Estudos têm associado a COVID-19 como uma complementopatia (Chatzidionysiou, Svenungsson \& Faustini, 2020), incluindo a identificação de vários componentes do sistema complemento no tecido pulmonar de pacientes com COVID-19 (Ahmad, Boschi-pinto \& Lopez, 2001).

Artigos têm abordado os estudos da chamada tempestade de citocina, uma reação inflamatória intensa, a qual tem seus produtos do sistema imune (fator de necrose tumoral (TNF); a IL-1 $\beta$; e a IL-6) associados ao estado de hipercoagulabilidade (Ackermann et al., 2020; Jose \& Manuel, 2020; Iba et al. 2020). Além disso, em pacientes com doença grave, os níveis de IL-6 foram superiores em pacientes que morreram, se comparados aos pacientes que sobreviveram (Zhou et al., 2020b), o que reforça, em certa medida, a tese de mortalidade aumentada em pacientes com tempestade de citocinas.

\section{Idade e a mortalidade}

Essa revisão apresentou a média de idade de 69,7 anos. Nesse trabalho aproximadamente $70 \%$ dos pacientes mortos por COVID-19 se encontram entre 60 e 89 anos.

Esses dados são reforçados por estudo em que a mediana das idades dos pacientes falecidos foi de 68 anos, entre os falecidos desse trabalho $83 \%$ tinha mais do que 60 anos ${ }^{(44)}$. Também nesse sentido, outro estudo mostrou que pacientes com idade avançada têm morrido 6,5 vezes mais do que os adultos jovens (OR =6,45; 95\% CI 3,86-10,77) (Lu et al., 2020).

Outro trabalho acompanhou dois grupos de macacos do gênero Rhesus, um grupo de macacos jovens, e outro de macacos velhos; e foi observado que os macacos velhos tinham manifestações graves do COVID-19 mais frequentemente se comparados com os macacos jovens (Yu et al., 2020). 
Além da alta mortalidade de pacientes velhos, outro dado que chama a atenção é a baixa mortalidade de pacientes jovens, visto que infecções respiratórias virais normalmente são letais tanto para população velha, quanto para a mais jovem, porém, por razões ainda não plenamente esclarecidas, a COVID-19 não se apresentou tão fatal em pacientes muito jovens nos primeiros meses da pandemia (Kang \& Jung, 2020).

\section{Trombos e DAD}

O padrão histopatológico melhor caracterizado da Síndrome da Angústia Respiratória Grave (SARS) é o dano alveolar difuso (DAD), apresentação extrema de lesão pulmonar aguda (Cheung, Graziano \& Smith, 2017), cujos aspectos estão descritos no Quadro 1. Os achados de DAD não possuem etiologia específica, sendo identificados na infecção pelo vírus influenza A subtipo H1N1, na Síndrome respiratória coronavírus do Oriente Médio (MERS) e na COVID-19 (Cheung, Graziano \& Smith, 2017; Alsaad et al., 2018; Ackermann et al., 2020). O presente trabalho observou DAD em 90,7\% das necrópsias avaliadas, o que evidência ser esta a apresentação histológica mais comum na COVID-19. O achado de DAD, com 75\% de ocorrência, também foi o mais comumente observado em outra revisão sistemática, que incluiu análises histológicas ante e postmortem de pulmões (Polak, Van Gool, Cohen, Von der Thüsen \& Van Paassen, 2020). Já os achados relacionados a macro e/ou microtrombos estavam presentes em $63,0 \%$ das necrópsias, demonstrando que parte dos pacientes que progridem para o óbito (26,9\% no presente trabalho) não desenvolvem trombos apesar da presença de DAD. A minoria das necrópsias (9,3\%) não demonstrou DAD e apresentou menor possibilidade de desenvolvimento de trombos (um paciente no presente estudo). Entre as análises histológicas que apresentavam trombos, 98,7\% estavam associadas ao DAD, sugerindo que a formação de trombos na COVID-19 esteja associada ao DAD possivelmente devido alteração vascular. Vale destacar que os achados de DAD, selecionados no presente trabalho por serem diretamente relacionados à SARS visando verificar a sua associação com a formação de trombos pulmonares, não são os únicos identificados nas necrópsias. No entanto ainda restam dúvidas acerca da patogenia desse processo, se poderia ser causado por lesão endotelial viral direta ou como resultado de tempestade de citocinas, choque, sepse viral.

\section{Trombos e sexo}

A maior quantidade de necrópsias realizadas em homens condiz com a descrição prévia acerca do maior risco de óbito em pacientes do sexo masculino na COVID-19 (Ye et al., 2020). Além disso, os achados do presente estudo demonstram que os pacientes do sexo masculino desenvolveram trombos e/ou microtrombos associados à COVID-19 com maior frequência que pacientes do sexo feminino.

Diante da previamente constatada diferença de letalidade entre homens e mulheres (Global Health 50/50, 2020), estudos sugerem como possíveis causas as distintas respostas imunes entre os sexos, a maior quantidade de receptores da enzima conversora de angiotensina 2 (ECA-2), fundamental para a entrada do SARS-CoV-2 nas células, em homens e idosos (Gebhard, Regitz-Zagrosek, Neuhauser, Morgan \& Klein, 2020) e a modulação sobre resposta inflamatória exercida pelo estradiol (Breithaupt-Faloppa, Correia, Prado, Stilhano, Ureshino \& Moreira, 2020).

\section{Trombos e comorbidades}

A COVID-19 criou um alerta sobre as enfermidades crônicas e sua elevada prevalência na população, assim, gerando a necessidade de reafirmar a importância não apenas do tratamento adequado para essas patologias, mas, sobretudo, para sua prevenção. Na presente revisão, evidenciou-se uma conexão entre a formação de trombos e a prevalência das seguintes comorbidades, respectivamente: TVP, hipertensão arterial, diabetes, DPOC, obesidade e tabagismo. 
Assim, a TVP pode induzir à tromboembolia pulmonar devido ao transporte do trombo da vasculatura profunda até atingir os pulmões (Osman, Ju, Sun \& Qi, 2018), situação que, quando não tratada com anticoagulantes, pode ser fatal (Kesieme, Kesieme, Jebbin, Irekpita \& Dongo, 2000). Entre os 119 pacientes analisados, sete apresentaram TVP na necrópsia, indicando uma alta incidência do distúrbio entre indivíduos contaminados pela COVID-19, cinco dentre eles mostraram também evidências de embolia pulmonar que, em um dos casos, constituiu causa direta da morte. Esse desenvolvimento destaca a importância do monitoramento de marcadores da coagulação e eventual aplicação de tratamentos anticoagulantes entre infectados pelo SARS-CoV-2 (Tchacil et al., 2020).

Ademais, dentre a presente amostra, $68,1 \%$ dos pacientes acometidos pela hipertensão arterial apresentaram trombos no pulmão quando necropsiados. Há evidências de que a hipertensão gera anormalidades nos componentes da tríade de Virchow (paredes vasculares, constituintes do sangue e fluxo sanguíneo), gerando um estado de hipercoagulabilidade (prótrombótico) (Lip \& Blann, 2000). Outros fatores de risco, tais como diabetes e tabagismo, também contribuiriam para este desenvolvimento (Lip, 2000) em consonância com os achados da presente revisão.

O diabetes também mostrou correlação com a apresentação de trombos, 64,1\% dos pacientes com diabetes da presente amostra apresentaram trombos na necrópsia. Há indicações de que a hiperglicemia comum no diabetes, além de facilitar a entrada do SARS-CoV-2 nas células, quando aguda, pode provocar disfunção endotelial e formação trombótica (Ceriello et al., 1993; Ceriello et al., 2020). Além disso, outro estudo mostrou que há uma menor taxa de sobrevivência em pacientes com diabetes em comparação com pacientes sem a doença e que, entre pacientes com diabetes e com a forma severa da COVID-19, homens eram mais propensos a morrer, corroborando os achados do presente trabalho (Yan et al., 2020b).

Entre os indivíduos afetados pela obesidade incluídos, 60\% apresentaram trombos, destacando uma possível correlação entre as patologias. Estudo realizado com amostra infectada por Francisella telarensis, doença que também causa uma tempestade de citocinas, indicou que a obesidade potencializa tal reação por ativar as mesmas vias de sinalização inflamatórias (Ramos et al., 2018). Ademais, indivíduos obesos naturalmente apresentam elevadas concentrações de leptina, hormônio pró-inflamatório com efeitos sistêmicos (La \& Matarese, 2004; Paz-Filho, Mastronardi, Franco, Wang, Wong \& Licinio, 2004). A partir de tais informações é possível teorizar que a obesidade contribui para um estado de inflamação crônica agudizado pela COVID-19 que, por consequência, é propenso a formação de trombos (Esmon, 2005).

\section{Trombos e achados macroscópicos}

Dentre os 119 indivíduos incluídos na presente amostra, apenas cinco $(4,2 \%)$ reportaram embolia pulmonar como complicação dentro do quadro da COVID-19. Outros estudos, no entanto, apresentaram incidências bem maiores, com cerca de 25\% dos pacientes com COVID-19 desenvolvendo embolia pulmonar (Helms et al., 2020; Klok et al., 2020). Tal discrepância chama atenção para este desdobramento da doença e necessita mais análises para elucidação.

\section{Trombos e hospitalização}

No presente estudo, constatou-se uma mediana de tempo de hospitalização de 6 dias. Na análise de dados desse trabalho, percebe-se no Gráfico 3 que o aparecimento de macrotrombos aumenta sua incidência nos pacientes que vieram a óbito gradativamente até o $10^{\circ}$ dia de hospitalização. Esses valores estão em acordo com trabalho que apresentou que a mediana do tempo de hospitalização de pacientes que vieram a óbito foi de 7,5 dias; em contrapartida, os pacientes que sobreviveram apresentaram uma mediana no tempo de hospitalização de 12,0 dias (Zhou et al., 2020a). Outro estudo também apresentou que a mediana do tempo de hospitalização de pacientes que sobreviveram foi de 13 dias (Wu, Hou, Liu, Chen \& Zhong, 2020), reforçando a ideia de que os pacientes que sobrevivem passam mais tempo internados. 
Identificou-se que pacientes que evoluíram para óbito tiveram níveis de D-dímero muito maiores se comparados aos de pacientes que sobreviveram, além disso, a concentração de D-dímero cresceu mais ou menos constantemente em pacientes incluídos no artigo, e permaneceu constantemente baixa em pacientes que sobreviveram (Zhou et al., 2020a).

Os níveis de IL-6 e ferritina também se mostraram maiores em pacientes que evoluíram para óbito se comparados a pacientes que não morreram após hospitalização (Zhou et al., 2020a). Esse achado também contribui para a ideia de que os pacientes que morrem pela COVID-19 têm relação com um processo inflamatório muito intenso. A IL-6, por exemplo, tem sido descrita como um dos constituintes da tempestade de citocinas que acomete alguns dos pacientes (Jose \& Manuel, 2020).

Levando-se em conta que os níveis de D-dímero e IL-6 aumentaram ao longo dos dias de hospitalização em pacientes que vieram a óbito, pode-se aventar a hipótese de que ocorre uma piora gradual do indivíduo, que tem maiores chances de falecer nos primeiros 12 dias de hospitalização (Zhou et al., 2020a), porém são necessários mais estudos envolvendo tempo de hospitalização e efeitos trombóticos em pacientes com covid-19 para se confirmar essa hipótese.

\section{Terapia anticoagulante}

Diante da observação de que os pacientes que vêm a óbito por COVID-19 apresentam níveis elevados dos produtos de degradação da fibrina e de D-dímero, além de tempos de protrombina e de tromboplastina parcial ativado prolongados (Tang, Li, Wang \& Sun, 2020), passou a ser utilizada a terapia anticoagulante no tratamento da doença.

Trabalho com 2773 pacientes hospitalizados, apesar de não ter especificado quais anticoagulantes foram utilizados nem ter identificado grande diferença nas taxas de mortalidade entre o grupo que recebeu tratamento anticoagulante (TAC) e o que não recebeu de modo geral durante a hospitalização, observou que o TAC profilático reduziu a necessidade de ventilação mecânica. Além disso, para o grupo que necessitou de ventilação mecânica, o uso de TAC foi associado a uma taxa de mortalidade de 29,1\% em comparação com 62,7\% para os pacientes que não receberam TAC (Paranjpe et al., 2020). Dessa forma, o TAC profilático seria uma importante ferramenta na redução da letalidade dos pacientes hospitalizados.

No entanto, entre os trabalhos da presente revisão que identificaram trombos, um citou o uso de TAC ou terapia antiagregante em doses profiláticas em todos os 11 pacientes (Lax et al., 2020) e outro artigo relatou que onze pacientes também fizeram uso de terapia anticoagulante antes do óbito (Menter et al., 2020). Entre as necrópsias que não apresentaram trombo e/ou microtrombos, citou-se uso de TAC em apenas um dos pacientes, cujo D-dímero estava elevado (Sekulic et al., 2020). Dessa forma, percebe-se que D-dímero elevado não é determinante para a formação de trombos e que mais estudos são necessários para verificar um melhor manejo do estado pró-trombótico dos pacientes com COVID-19.

\section{Conclusão}

Nessa revisão sistemática, a maioria das necrópsias evidenciou trombos nos pulmões. Pacientes com microtrombos têm maior chance de vir a óbito nos primeiros 9 dias de internação. $\mathrm{O}$ achado microscópico mais presente foi compatível com dano alveolar difuso; pacientes do sexo masculino que vieram a óbito desenvolveram mais macro e/ou microtrombos que aqueles do sexo feminino. Comorbidades como TVP, hipertensão e diabetes foram associados a maior incidência de trombos. As limitações desse estudo incluem o número relativamente baixo de necrópsias, o que se deve ao fato de muitos protocolos de segurança limitarem o manejo de cadáveres mortos com COVID-19 além da avaliação acerca de trombos ter sido feita apenas em pacientes que vieram a óbito, limitando a compreensão dessa intercorrência em pacientes com COVID-19 de forma geral. Mais estudos precisam ser realizados visando associar os achados necroscópicos pulmonares à fisiopatogenia da enfermidade e o uso de terapia anticoagulante com COVID-19. 
Assim, tendo em vista a quantidade limitada de necrópsias disponíveis para esse trabalho, faz-se necessário novos estudos que visem explicar a associação entre tempo de hospitalização e formação de trombos e estágios do DAD. Além de ensaios clínicos randomizados que mensurem terapia por anticoagulante em pacientes já internados.

\section{Referências}

Ackermann, M., Verleden, S. E., Kuehnel, .M, Haverich, A., Welte, T., Laenger, F., et al. (2020). Pulmonary Vascular Endothelialitis, Thrombosis, and Angiogenesis in Covid-19. N Engl J Med [Internet], NEJMoa2015432. http://www.nejm.org/doi/10.1056/NEJMoa2015432

Adachi, T., Chong, J. M., Nakajima, N., Sano, M., Yamazaki, J., Miyamoto, I., Nishioka, H., Akita, H., Sato, Y., Kataoka, M., Katano, H., Tobiume, M., Sekizuka, T., Itokawa, K., Kuroda, M., \& Suzuki, T. (2020). Clinicopathologic and Immunohistochemical Findings from Autopsy of Patient with COVID-19, Japan. Emerging infectious diseases, 26(9), 2157-2161. https://doi.org/10.3201/eid2609.201353

Aguiar, D., Lobrinus, J. A., Schibler, M., Fracasso, T., \& Lardi, C. (2020). Inside the lungs of COVID-19 disease. International journal of legal medicine, 134(4), 1271-1274. https://doi.org/10.1007/s00414-020-02318-9

Ahmad, O. B., Boschi-pinto, C., \& Lopez, A. D. (2001). Age standardization of rates: a new WHO standard. GPE Discuss Pap Ser., (31), 1-14.

Alsaad, K. O., Hajeer, A. H., Al Balwi, M., Al Moaiqel, M., Al Oudah, N., Al Ajlan, A., AlJohani, S., Alsolamy, S., Gmati, G. E., Balkhy, H., Al-Jahdali, H. H., Baharoon, S. A., \& Arabi, Y. M. (2018). Histopathology of Middle East respiratory syndrome coronovirus (MERS-CoV) infection - clinicopathological and ultrastructural study. Histopathology, 72(3), 516-524. https://doi.org/10.1111/his.13379

Barth, R. F., Xu, X., \& Buja, L. M. (2020). A Call to Action: The Need for Autopsies to Determine the Full Extent of Organ Involvement Associated With COVID-19. Chest, 158(1), 43-44. https://doi.org/10.1016/j.chest.2020.03.060

Barton, L. M., Duval, E. J., Stroberg, E., Ghosh, S., \& Mukhopadhyay, S. (2020). COVID-19 Autopsies, Oklahoma, USA. Am J Clin Pathol., 153(6), 725-33.

Breithaupt-Faloppa, A. C., Correia, C. J., Prado, C. M., Stilhano, R. S., Ureshino, R. P., \& Moreira L. F. P. (2020). 17ß-Estradiol, a potential ally to alleviate SARS-CoV-2 infection. Clinics (Sao Paulo), 75, e1980.

Buja, L. M., Wolf, D., Zhao, B., Akkanti, B., McDonald, M., Lelenwa, L., et al. (2020). The emerging spectrum of cardiopulmonary pathology of the coronavirus disease 2019 (COVID-19): Report of 3 autopsies from Houston, Texas, and review of autopsy findings from other United States cities. Cardiovasc Pathol [Internet], 48, 107233. https://doi.org/10.1016/j.carpath.2020.107233

Carsana, L., Sonzogni, A., Nasr, A., Rossi, R. S., Pellegrinelli, A., Zerbi, P., et al. (2020). Pulmonary post-mortem findings in a series of COVID-19 cases from northern Italy: a two-centre descriptive study. Lancet Infect Dis [Internet], 0(0), 6-11. https://linkinghub.elsevier.com/retrieve/pii/S1473309920304345

Ceriello A. (1993). Coagulation activation in diabetes mellitus: the role of hyperglycaemia and therapeutic prospects. Diabetologia, 36(11), 1119-1125. https://doi.org/10.1007/BF00401055

Ceriello, A., Standl, E., Catrinoiu, D., Itzhak, B., Lalic, N. M., Rahelic, D., Schnell, O., Škrha, J., Valensi, P., \& Diabetes and Cardiovascular Disease (D\&CVD) EASD Study Group (2020). Issues of Cardiovascular Risk Management in People With Diabetes in the COVID-19 Era. Diabetes care, 43(7), 1427-1432. https://doi.org/10.2337/dc20-0941

Chatzidionysiou, K., Svenungsson, E., \& Faustini, F. (2020). Could severe COVID-19 be considered a complementopathy?. Lupus science \& medicine, 7(1), e000415. https://doi.org/10.1136/lupus-2020-000415

Chen, T., Wu, D., Chen, H., Yan, W., Yang, D., Chen, G., et al. (2020). Clinical characteristics of 113 deceased patients with coronavirus disease 2019: Retrospective study. BMJ, 368(December 2019), 1-14.

Cheung, O. Y., Graziano, P., \& Smith, M. L. (2017). Acute Lung Injury. In: Practical Pulmonary Pathology: A Diagnostic Approach A Volume in the Pattern Recognition Series, p. 125-46.

Craver, R., Huber, S., Sandomirsky, M., McKenna, D., Schieffelin, J., \& Finger, L. (2020). Fatal Eosinophilic Myocarditis in a Healthy 17-Year-Old Male with Severe Acute Respiratory Syndrome Coronavirus 2 (SARS-CoV-2c). Fetal and pediatric pathology, $39(3), 263-268$. https://doi.org/10.1080/15513815.2020.1761491

Da Costa, M. G., Poppelaars, F., Van Kooten, C., Mollnes, T. E., Tedesco, F., Würzner, R., et al. (2018). Age and sex-associated changes of complement activity and complement levels in a healthy caucasian population. Front Immunol., 9(NOV).

Esmon, C. T. (2005). The interactions between inflammation and coagulation. Br J Haematol., 131(4), 417-30.

Farkash, E. A., Wilson, A. M., \& Jentzen, J. M. (2020). Ultrastructural Evidence for Direct Renal Infection with SARS-CoV-2. Journal of the American Society of Nephrology : JASN, 31(8), 1683-1687. https://doi.org/10.1681/ASN.2020040432

Fox, S. E., Akmatbekov, A., Harbert, J. L., Li, G., Quincy Brown, J, \& Vander Heide, R. S. (2020). Pulmonary and cardiac pathology in African American patients with COVID-19: an autopsy series from New Orleans. Lancet Respir Med [Internet], (January), 19-21. https://linkinghub.elsevier.com/retrieve/pii/S2213260020302435

Gebhard, C., Regitz-Zagrosek, V., Neuhauser, H. K., Morgan, R., \& Klein, S. L. (2020). Impact of sex and gender on COVID-19 outcomes in Europe. Biology of sex differences, 11(1), 29. https://doi.org/10.1186/s13293-020-00304-9

Gorbalenya, A. E., Baker, S. C., Baric, R. S., de Groot, R. J., Drosten, C., Gulyaeva, A. A., et al. (2020). The species Severe acute respiratory syndrome- 
related coronavirus: classifying 2019-nCoV and naming it SARS-CoV-2. Nat Microbiol., 5(4), 536-44.

Guan, W., Ni, Z., Hu, Y., Liang, W., Ou, C., He, J., et al. (2020). Clinical characteristics of coronavirus disease 2019 in China. N Engl J Med., 382(18), 170820 .

Helms, J., Tacquard, C., Severac, F., Leonard-Lorant, I., Ohana, M., Delabranche, X., Merdji, H., Clere-Jehl, R., Schenck, M., Fagot Gandet, F., Fafi-Kremer, S., Castelain, V., Schneider, F., Grunebaum, L., Anglés-Cano, E., Sattler, L., Mertes, P. M., Meziani, F., \& CRICS TRIGGERSEP Group (Clinical Research in Intensive Care and Sepsis Trial Group for Global Evaluation and Research in Sepsis) (2020). High risk of thrombosis in patients with severe SARS-CoV-2 infection: a multicenter prospective cohort study. Intensive care medicine, 46(6), 1089-1098. https://doi.org/10.1007/s00134-020-06062-x

Huang, C., Wang, Y., Li, X., Ren, L., Zhao, J., Hu, Y., et al. (2020). Clinical features of patients infected with 2019 novel coronavirus in Wuhan, China. Lancet., 395(10223), 497-506.

Iba, T., Levy, J. H., Levi, M., Connors, J. M., \& Thachil, J. (2020). Coagulopathy of Coronavirus Disease 2019. Critical care medicine, 48(9), 1358-1364. https://doi.org/10.1097/CCM.0000000000004458

Iba, T., Levy, J. H., Connors, J. M., Warkentin, T. E., Thachil, J., \& Levi, M. (2020). The unique characteristics of COVID-19 coagulopathy. Crit Care., 24(1), 360 .

Jin, H., Hong, C., Chen, S., Zhou, Y., Wang, Y., Mao, L, et al. (2020). Consensus for prevention and management of coronavirus disease 2019 (COVID-19) for neurologists. Stroke Vasc Neurol., (M), 1-6.

Jose, R. J., \& Manuel, A. (2020). COVID-19 cytokine storm: the interplay between inflammation and coagulation. Lancet Respir Med., 8(6), e46-7.

Kang, S. J., \& Jung, S. I. (2020). Age Related Morbidity and Mortality among Patients with COVID-19. Infect Chemother, 52(2), 154-64.

Kang, Y., Chen, T., Mui, D., Ferrari, V., Jagasia, D., Scherrer-Crosbie, M., et al. (2020). Cardiovascular manifestations and treatment considerations in covid19. Heart., 2, 1-10.

Karami, P., Naghavi, M., Feyzi, A., Aghamohammadi, M., Novin, M. S., Mobaien, A., Qorbanisani, M., Karami, A., \& Norooznezhad, A. H. (2020). WITHDRAWN: Mortality of a pregnant patient diagnosed with COVID-19: A case report with clinical, radiological, and histopathological findings. Travel medicine and infectious disease, 101665. https://doi.org/10.1016/j.tmaid.2020.101665

Kesieme, E., Kesieme, C., Jebbin, N., Irekpita, E., \& Dongo, A. (2011). Deep vein thrombosis: a clinical review. Journal of blood medicine, 2, 59-69. https://doi.org/10.2147/JBM.S19009

Klok, F. A., Kruip, M., van der Meer, N., Arbous, M. S., Gommers, D., Kant, K. M., Kaptein, F., van Paassen, J., Stals, M., Huisman, M. V., \& Endeman, H. (2020). Incidence of thrombotic complications in critically ill ICU patients with COVID-19. Thrombosis research, 191 , 145-147. https://doi.org/10.1016/j.thromres.2020.04.013

Konopka, K. E., Wilson, A., \& Myers, J. L. (2020). Postmortem Lung Findings in a Patient With Asthma and Coronavirus Disease 2019. Chest, 158(3), e99e101. https://doi.org/10.1016/j.chest.2020.04.032

La Cava, A., \& Matarese, G. (2004). The weight of leptin in immunity. Nature reviews. Immunology, 4(5), 371-379. https://doi.org/10.1038/nri1350

Lacy, J. M., Brooks, E. G., Akers, J., Armstrong, D., Decker, L., Gonzalez, A., Humphrey, W., Mayer, R., Miller, M., Perez, C., Arango, J., Sathyavagiswaran, L., Stroh, W., \& Utley, S. (2020). COVID-19: Postmortem Diagnostic and Biosafety Considerations. The American journal of forensic medicine and pathology, 41(3), 143-151. https://doi.org/10.1097/PAF.0000000000000567

Lax, S. F., Skok, K., Zechner, P., Kessler, H. H., Kaufmann, N., Koelblinger, C., et al. (2020). Pulmonary Arterial Thrombosis in COVID-19 With Fatal Outcome: Results From a Prospective, Single-Center, Clinicopathologic Case Series. Ann Intern Med [Internet], M20-2566. https://www.acpjournals.org/doi/10.7326/M20-2566

Lip, G. Y. (2000). Hypertension and the prothrombotic state. Journal of human hypertension, 14(10-11), 687-690. https://doi.org/10.1038/sj.jhh.1001051

Lip, G. Y., \& Blann, A. D. (2000). Does hypertension confer a prothrombotic state? Virchow's triad revisited. Circulation, 101(3), 218-220. https://doi.org/10.1161/01.cir.101.3.218

Lu, L., Zhong, W., Bian, Z., Li, Z., Zhang, K., Liang, B., Zhong, Y., Hu, M., Lin, L., Liu, J., Lin, X., Huang, Y., Jiang, J., Yang, X., Zhang, X., \& Huang, Z. (2020). A comparison of mortality-related risk factors of COVID-19, SARS, and MERS: A systematic review and meta-analysis. The Journal of infection, 81(4), e18-e25. https://doi.org/10.1016/j.jinf.2020.07.002

Magro, C., Mulvey, J. J., Berlin, D., Nuovo, G., Salvatore, S., Harp, J., Baxter-Stoltzfus, A., \& Laurence, J. (2020). Complement associated microvascular injury and thrombosis in the pathogenesis of severe COVID-19 infection: A report of five cases. Translational research : the journal of laboratory and clinical medicine, 220, 1-13. https://doi.org/10.1016/j.trs1.2020.04.007

Menter, T., Haslbauer, J. D., Nienhold, R., Savic, S., Hopfer, H., Deigendesch, N., Frank, S., Turek, D., Willi, N., Pargger, H., Bassetti, S., Leuppi, J. D., Cathomas, G., Tolnay, M., Mertz, K. D., \& Tzankov, A. (2020). Postmortem examination of COVID-19 patients reveals diffuse alveolar damage with severe capillary congestion and variegated findings in lungs and other organs suggesting vascular dysfunction. Histopathology, 77(2), 198-209. https://doi.org/10.1111/his.14134

Moher, D., Liberati, A., Tetzlaff, J., Altman, D. G., \& PRISMA Group (2009). Preferred reporting items for systematic reviews and meta-analyses: the PRISMA statement. PLoS medicine, 6(7), e1000097. https://doi.org/10.1371/journal.pmed.1000097

Nunes Duarte-Neto, A., de Almeida Monteiro, R. A., da Silva, L. F. F., Malheiros, D. M. A. C., de Oliveira, E. P., Theodoro Filho, J., et al. (2020). Pulmonary and systemic involvement of COVID-19 assessed by ultrasound-guided minimally invasive autopsy. Histopathology [Internet], 0-2. http://www.ncbi.nlm.nih.gov/pubmed/32443177

Osman, A. A., Ju, W., Sun, D., \& Qi, B. (2018). Deep venous thrombosis: a literature review. Int J Clin Exp Med., 11(3), 1551-61. 
Paranjpe, I., Fuster, V., Lala, A., Russak, A. J., Glicksberg, B. S., Levin, M. A., Charney, A. W., Narula, J., Fayad, Z. A., Bagiella, E., Zhao, S., \& Nadkarni, G. N. (2020). Association of Treatment Dose Anticoagulation With In-Hospital Survival Among Hospitalized Patients With COVID-19. Journal of the American College of Cardiology, 76(1), 122-124. https://doi.org/10.1016/j.jacc.2020.05.001

Paz-Filho, G., Mastronardi, C., Franco, C. B., Wang, K. B., Wong, M. L., \& Licinio, J. (2012). Leptin: molecular mechanisms, systemic pro-inflammatory effects, and clinical implications. Arq Bras Endocrinol Metabol., 56(9), 597-607.

Pei, G., Zhang, Z., Peng, J., Liu, L., Zhang, C., Yu, C., et al. (2020). Renal Involvement and Early Prognosis in Patients with COVID-19 Pneumonia. J Am Soc Nephrol., 2019, 1-9.

Polak, S. B., Van Gool, I. C., Cohen, D., von der Thüsen, J. H., \& van Paassen, J. (2020). A systematic review of pathological findings in COVID-19: a pathophysiological timeline and possible mechanisms of disease progression. Modern pathology : an official journal of the United States and Canadian Academy of Pathology, Inc, 33(11), 2128-2138. https://doi.org/10.1038/s41379-020-0603-3

Ramos Muniz, M. G., Palfreeman, M., Setzu, N., Sanchez, M. A., Saenz Portillo, P., Garza, K. M., Gosselink, K. L., \& Spencer, C. T. (2018). Obesity Exacerbates the Cytokine Storm Elicited by Francisella tularensis Infection of Females and Is Associated with Increased Mortality. BioMed research international, 2018, 3412732. https://doi.org/10.1155/2018/3412732

Schweitzer, W., Ruder, T., Baumeister, R., Bolliger, S., Thali, M., Meixner, E., \& Ampanozi, G. (2020). Implications for forensic death investigations from first Swiss post-mortem CT in a case of non-hospital treatment with COVID-19. Forensic Imaging, 21, 200378. https://doi.org/10.1016/j.fri.2020.200378

Sekulic, M., Harper, H., Nezami, B. G., Shen, D. L., Sekulic, S. P., Koeth A. T., et al. (2020). Molecular Detection of SARS-CoV-2 Infection in FFPE Samples and Histopathologic Findings in Fatal SARS-CoV-2 Cases. Am J Clin Pathol [Internet], 1-11. http://www.ncbi.nlm.nih.gov/pubmed/32451533

Sex, gender and COVID-19: overview and resources [Internet]. (2020). Global Health 50/50. https://globalhealth5050.org/covid19/

Tan, W., Zhao, X., Ma, X., Wang, W., Niu, P., Xu, W., et al. (2020). Notes from the Field A Novel Coronavirus Genome Identified in a Cluster of Pneumonia Cases - Wuhan, China 2019 - 2020. China CDC Wkly., 2(4), 61-2.

Tang, N., Li, D., Wang, X., \& Sun, Z. (2020). Abnormal coagulation parameters are associated with poor prognosis in patients with novel coronavirus pneumonia. Journal of thrombosis and haemostasis : JTH, 18(4), 844-847. https://doi.org/10.1111/jth.14768

Thachil, J., Tang, N., Gando, S., Falanga, A., Cattaneo, M., Levi, M., Clark, C., \& Iba, T. (2020). ISTH interim guidance on recognition and management of coagulopathy in COVID-19. Journal of thrombosis and haemostasis : JTH, 18(5), 1023-1026. https://doi.org/10.1111/jth.14810

Varga, Z., Flammer, A. J., Steiger, P., Haberecker, M., Andermatt, R., Zinkernagel, A. S., Mehra, M. R., Schuepbach, R. A., Ruschitzka, F., \& Moch, H. (2020). Endothelial cell infection and endotheliitis in COVID-19. Lancet (London, England), 395(10234), 1417-1418. https://doi.org/10.1016/S01406736(20)30937-5

Wang, D., Hu, B., Hu, C., Zhu, F., Liu, X., Zhang, J., et al. (2020). Clinical Characteristics of 138 Hospitalized Patients with 2019 Novel Coronavirus-Infected Pneumonia in Wuhan, China. JAMA - J Am Med Assoc., 323(11), 1061-9.

Wichmann, D., Sperhake, J. P., Lütgehetmann, M., Steurer, S., Edler, C., Heinemann, A., Heinrich, F., Mushumba, H., Kniep, I., Schröder, A. S., Burdelski, C., de Heer, G., Nierhaus, A., Frings, D., Pfefferle, S., Becker, H., Bredereke-Wiedling, H., de Weerth, A., Paschen, H. R., Sheikhzadeh-Eggers, S., Kluge, S. (2020). Autopsy Findings and Venous Thromboembolism in Patients With COVID-19: A Prospective Cohort Study. Annals of internal medicine, 173(4), 268277. https://doi.org/10.7326/M20-2003

World Health Organization (WHO). (2020). Coronavirus disease (COVID-19): Situation Report - 162 https://www.who.int/docs/defaultsource/coronaviruse/20200630-covid-19-sitrep-162.pdf?sfvrsn=e00a5466_2

Wu, Y., Hou, B., Liu, J., Chen, Y., \& Zhong, P. (2020). Risk Factors Associated With Long-Term Hospitalization in Patients With COVID-19: A SingleCentered, Retrospective Study. Frontiers in medicine, 7, 315. https://doi.org/10.3389/fmed.2020.00315

Xie, M., \& Chen, Q. (2020). Insight into 2019 novel coronavirus — An updated interim review and lessons from SARS-CoV and MERS-CoV. Int J Infect Dis [Internet], 94, 119-24. https://doi.org/10.1016/j.ijid.2020.03.071

Yan, L., Mir, M., Sanchez, P., Beg, M., Peters, J., Enriquez, O., \& Gilbert, A. (2020a). COVID-19 in a Hispanic Woman. Archives of pathology \& laboratory medicine, 144(9), 1041-1047. https://doi.org/10.5858/arpa.2020-0217-SA

Yan, Y., Yang, Y., Wang, F., Ren, H., Zhang, S., Shi, X., et al. (2020b). Clinical characteristics and outcomes of patients with severe covid-19 with diabetes. BMJ Open Diabetes Res Care., 8(1), 1-9.

Ye, Q., Wang, B., Mao, J., Fu, J., Shang, S., Shu, Q., \& Zhang, T. (2020). Epidemiological analysis of COVID-19 and practical experience from China. Journal of medical virology, 92(7), 755-769. https://doi.org/10.1002/jmv.25813

Yoshikawa, A., Fukuoka, J., \& Andrey, B. (2018). Acute respiratory distress syndrome (ARDS) / diffuse alveolar damage (DAD). [Internet]. PathologyOutlines.com website http://www.pathologyoutlines.com/topic/lungnontumordiffusealveolardamage.html

Yu, P., Qi, F., Xu, Y., Li, F., Liu, P., Liu, J., et al. (2020). Age-related rhesus macaque models of COVID-19. Anim Model Exp Med., 3(1), 93-7.

Zhou, F., Yu, T., Du, R., Fan, G., Liu, Y., Liu, Z., et al. (2020a). Clinical course and risk factors for mortality of adult inpatients with COVID-19 in Wuhan, China: a retrospective cohort study. Lancet., 395(10229), 1054-62.

Zhou, Y., Li, W., Wang, D., Mao, L., Jin, H., Li, Y., et al. (2020b). Clinical time course of COVID-19, its neurological manifestation and some thoughts on its management. Stroke Vasc Neurol., svn-2020-000398.

Zhu, N., Zhang, D., Wang, W., Li, X., Yang, B., Song, J., et al. (2020). A novel coronavirus from patients with pneumonia in China, 2019. N Engl J Med., 382(8), 727-33. 